\title{
Immune reprogramming via PD-1 inhibition enhances early-stage lung cancer survival
}

Geoffrey J. Markowitz, ${ }^{1,2,3}$ Lauren S. Havel, , ${ }^{1,2,3}$ Michael J.P. Crowley, ${ }^{3,4}$ Yi Ban, ${ }^{1,2,3}$ Sharrell B. Lee, ${ }^{1,3}$ Jennifer S. Thalappillil, ${ }^{1,3}$ Navneet Narula, ${ }^{5}$ Bhavneet Bhinder, ${ }^{6}$ Olivier Elemento, ${ }^{6}$ Stephen T.C. Wong, ${ }^{7}$ Dingcheng Gao, ${ }^{1,2,3}$ Nasser K. Altorki, ${ }^{1,3}$ and Vivek Mittal ${ }^{1,2,3}$

'Department of Cardiothoracic Surgery, ${ }^{2}$ Department of Cell and Developmental Biology, and ${ }^{3}$ Neuberger Berman Lung Cancer Center, Weill Cornell Medicine, New York, New York, USA. ${ }^{4}$ Weill Cornell Graduate School of Medical Sciences, New York, New York, USA. ${ }^{5}$ Department of Pathology, Weill Cornell Medicine, New York, New York, USA. ${ }^{6}$ Caryl and Israel Englander Institute for Precision Medicine, HRH Prince Alwaleed Bin Talal Bin Abdulaziz Alsaud Institute for Computational Biomedicine, Department of Physiology and Biophysics, Weill Cornell Medicine, New York, New York, USA. ${ }^{7}$ Houston Methodist Research Institute, Houston, Texas, USA.

Success of immune checkpoint inhibitors in advanced non-small-cell lung cancer (NSCLC) has invigorated their use in the neoadjuvant setting for early-stage disease. However, the cellular and molecular mechanisms of the early immune responses to therapy remain poorly understood. Through an integrated analysis of early-stage NSCLC patients and a Kras mutant mouse model, we show a prevalent programmed cell death 1/programmed cell death 1 ligand 1 (PD-1/PD-L1) axis exemplified by increased intratumoral PD-1+ $T$ cells and PD-L1 expression. Notably, tumor progression was associated with spatiotemporal modulation of the immune microenvironment with dominant immunosuppressive phenotypes at later phases of tumor growth. Importantly, PD-1 inhibition controlled tumor growth, improved overall survival, and reprogrammed tumor-associated lymphoid and myeloid cells. Depletion of T lymphocyte subsets demonstrated synergistic effects of those populations on PD-1 inhibition of tumor growth. Transcriptome analyses revealed T cell subset-specific alterations corresponding to degree of response to the treatment. These results provide insights into temporal evolution of the phenotypic effects of PD-1/PD-L1 activation and inhibition and motivate targeting of this axis early in lung cancer progression.

Conflict of interest: The authors have declared that no conflict of interest exists.

Submitted: August 10, 2017

Accepted: May 23, 2018

Published: July 12, 2018

Reference information: JCI Insight. 2018;3(13):e96836. https://doi.org/10.1172/jci. insight. 96836.

\section{Introduction}

Lung cancer is the leading cause of cancer-related mortality worldwide, with an estimated 1.3 million new cases each year $(1,2)$. Non-small-cell lung cancer (NSCLC) constitutes approximately $80 \%$ of all lung cancer cases and has a 5 -year survival rate of only $15 \%-20 \%$ (3). Immune checkpoint inhibitors have begun to revolutionize the survival prospects of cancer patients (4-6), particularly those blocking the PD-1/PD-L1 (programmed cell death 1/programmed cell death 1 ligand 1) axis, which have yielded objective response rates of about $20 \%$ and are currently approved by the FDA for a subset of patients with advanced disease (7-9). Importantly, in patients with advanced NSCLC and PD-L1 expression on at least 50\% of tumor cells, PD-1 inhibition was associated with longer progression-free and overall survival than platinum-based chemotherapy (10). However, large cohorts of patients do not display a clinical response to PD-1/PD-L1 axis inhibition at late stages in disease progression. This has been attributed to several potential mechanisms, including low PD-L1 expression, T cell exclusion from tumor islets (cold tumors), and T cell dysfunction that emerges in the context of chronic antigen exposure (11-15). Approximately $20 \%-25 \%$ of the 220,000 patients diagnosed with NSCLC in the United States present potentially operable early-stage (I-IIIA) disease, and these numbers are likely to increase with the recent widespread implementation of CT screening for lung cancer (16-18). Early-stage disease presents with a more intact immune system and a lower tumor burden, possibly affording immune checkpoint blockade the potential to confer a more favorable outcome.

PD-1 is expressed on T cells during activation (19) and is further upregulated as T cells acquire dysfunctional characteristics $(4,20)$. Its regulation and activation have been shown to be critical negative regulators 
of functional antitumor immune responses, and PD-1 is now commonly accepted as a key marker of T cell dysfunctionality $(15,17)$. Mechanistically, in T cells, PD-1 inhibits TCR signaling, with subsequent suppressive effects on proliferation and cytokine production and increased cell death $(21,22)$. Inhibition of the PD-1/ PD-L1 axis via therapeutic anti-PD-1 and anti-PD-L1 antibodies interrupts the activity of this axis, leading to reinvigoration of $\mathrm{T}$ cell-mediated antitumor activity (4). Studies have primarily focused on the response of $\mathrm{CD}^{+} \mathrm{T}$ cells to therapeutic inhibition of the axis; however, recent data suggest that effects of treatment on other components of the microenvironment may also affect outcomes $(23,24)$. While the efficacy of inhibition of this axis is established in late stages in tumor progression, the cellular and molecular mechanisms underlying the evolving immune responses to therapy at early stages in disease progression are unknown.

We explored the expression and functional ramifications of targeting the PD-1/PD-L1 axis in early-stage NSCLC. In early-stage treatment-naive human NSCLC, we observed T cell infiltrates in tumor beds, with expression of PD-1 on both $\mathrm{CD}^{+}$and $\mathrm{CD} 8^{+} \mathrm{T}$ cells. Similar observations in the orthotopic model of Kras-driven NSCLC led us to test pharmacological inhibition of PD-1, which resulted in tumor control and improved survival in mice. PD-1 blockade increased proliferation of $\mathrm{T}$ lymphocytes, reprogrammed multiple elements of the immune microenvironment, and significantly enhanced effector functions of $\mathrm{CD}^{+} \mathrm{T}$ cells. Depletion of $\mathrm{CD}^{+}$and $\mathrm{CD} 8^{+} \mathrm{T}$ cells diminished efficacy of anti-PD-1 therapy, with strong synergistic effects between the two populations in modulating tumor growth. Gene expression was significantly altered in both $\mathrm{CD}^{+}$and $\mathrm{CD} 8^{+} \mathrm{T}$ cells in response to anti-PD-1 therapy, with differential variation in these subsets and response corresponding to the degree of antitumor efficacy. These results provide a mechanistic rationale for therapeutic intervention of the PD-1/PD-L1 axis in early-stage lung cancer.

\section{Results}

The immune-suppressive PD-1/PD-L1 axis is prevalent in early-stage NSCLC. To evaluate the status of the immune microenvironment at early stages in disease progression, we examined tumor tissue and matched adjacent nonneoplastic lungs from a cohort of stage I-IIIA treatment-naive NSCLC patients for T cell infiltration in tumor beds, PD-1 expression on $\mathrm{CD}^{+}$and $\mathrm{CD} 8^{+} \mathrm{T}$ cells, and PD-L1 abundance (Supplemental Table 1; supplemental material available online with this article; https://doi.org/10.1172/jci.insight.96836DS1). $\mathrm{H} \& \mathrm{E}$ staining and IHC analysis showed abundant $\mathrm{CD}^{+} \mathrm{T}$ cells infiltrating the tumor beds compared with matched adjacent nontumor tissue (Figure $1 \mathrm{~A}$ ). A low $\mathrm{CD}^{+}$to $\mathrm{CD} 4^{+} \mathrm{T}$ cell ratio was observed in the $\mathrm{CD}^{+} \mathrm{T}$ cells, as determined by flow cytometry (Figure 1B). We next evaluated expression of the inhibitory PD-1/PD-L1 axis and observed that a substantial proportion of tumor-infiltrating T cells expressed PD-1 (Figure 1B). Significantly enhanced proportions of $\mathrm{PD}-1^{+}$cells were observed in both the $\mathrm{CD} 8^{+}$and the $\mathrm{CD}^{+} \mathrm{T}$ cell compartments in the tumor tissue compared with adjacent tissue (Figure $1 \mathrm{~B}$ and Supplemental Figure 1). Mean fluorescent intensity analysis showed higher PD-1 expression in tumor-infiltrating $\mathrm{T}$ cells compared with adjacent tissue (Figure 1C). Tumor-infiltrating $\mathrm{CD}^{+} \mathrm{T}$ cells displayed a trend toward higher PD-1 expression than $\mathrm{CD}^{+} \mathrm{T}$ cells as a group; however, this higher expression was driven by a subset of samples showing substantially elevated PD-1 expression, while the majority of samples demonstrated similar PD-1 expression to that observed in the $\mathrm{CD}^{+} \mathrm{T}$ cells (Figure 1C). Next, we evaluated PD-L1 and observed increased PD-L1 expression in tumors compared with adjacent tissue, with some tumors showing PD-L1 expression on tumor-infiltrating $\mathrm{CD} 45^{+}$cells as well as transformed cells, suggesting that multiple cell types may mediate activation of the PD-1/PD-L1 axis (Figure 1D). Together, these data suggest prevalence and potential activation of the immune-suppressive PD-1/PD-L1 axis in early-stage NSCLC and provide a rationale for targeting early-stage tumors with anti-PD-1/anti-PD-L1 immunotherapy.

The HKP1 model exhibits PD-1/PD-L1 axis prevalence in early-stage NSCLC. We determined whether the PD-1/PD-L1 axis observed in early-stage NSCLC patients was also prevalent and active in a mouse model of NSCLC, as this would allow us to test the therapeutic efficacy of PD-1/PD-L1 inhibition and allow us to determine mechanisms of response in early NSCLC. We utilized the HKP1 ( $\left.\mathrm{Kras}^{\mathrm{G} 12 \mathrm{D}} \mathrm{p}^{53^{--}}\right)$orthotopic, immunocompetent, syngeneic preclinical model of NSCLC (25), which we had previously shown to exhibit histological similarities to human adenocarcinoma (25). We performed spatiotemporal analysis of the immune microenvironment in the HKP1 model as a function of tumor growth measured by bioluminescence imaging (Supplemental Figure 2). Similar to the findings in early-stage NSCLC patients, HKP1 tumors at early stages of progression (week 1 ) showed accumulation of infiltrating $\mathrm{CD}^{+} \mathrm{T}$ cells in the tumor bed (Figure 2A), low $\mathrm{CD}^{+}$to $\mathrm{CD}^{+} \mathrm{T}$ cell ratios (Figure $2 \mathrm{~B}$ ), and $\mathrm{PD}-1$ expression on $\mathrm{CD}^{+}$ and $\mathrm{CD}^{+} \mathrm{T}$ cells (Figure $2 \mathrm{C}$ and Supplemental Figure 3). Similar to observations in clinical samples, 
A

’
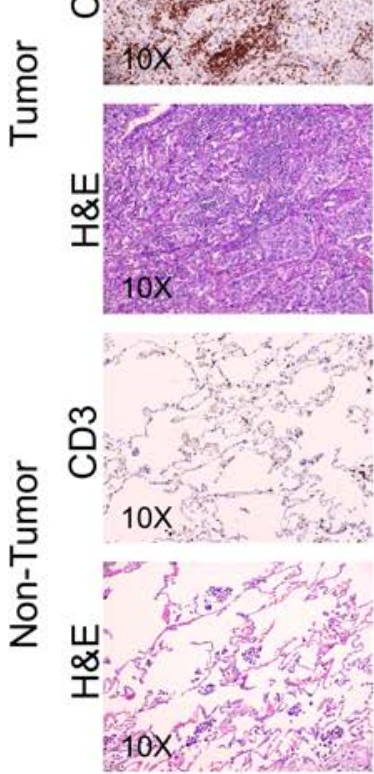

Patient 2

\section{Patient 3}
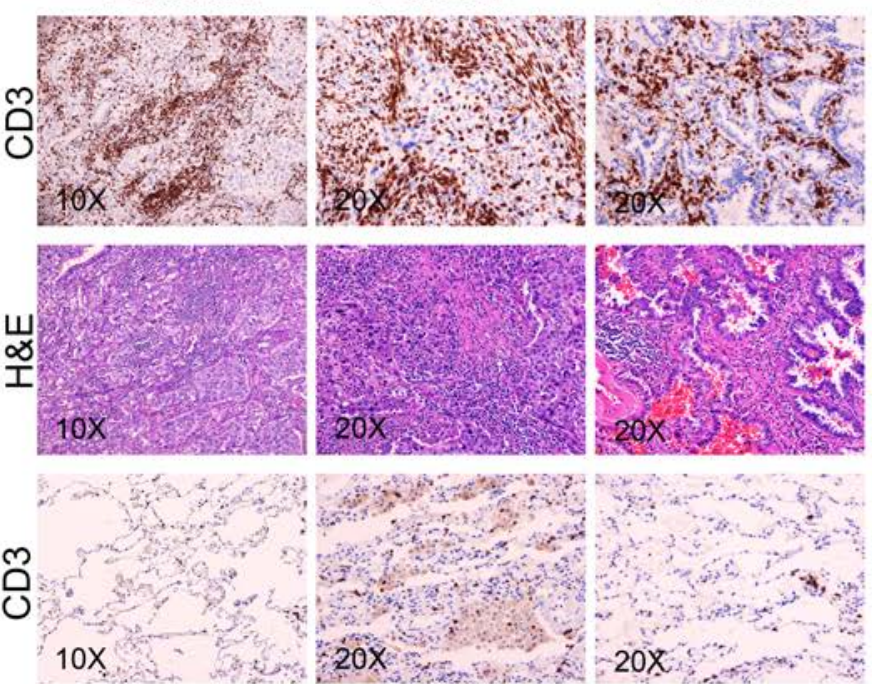

$20 \times \quad 20 \times-7$

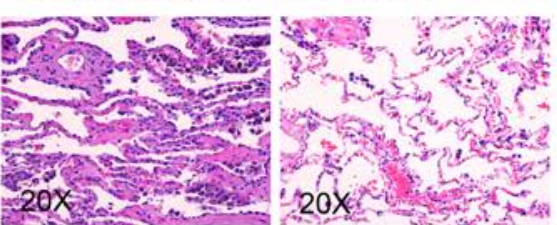

Figure 1. Prevalent PD-1/PD-L1 axis in early-stage human lung cancer patients. (A) Representative anti-CD3 IHC and $\mathrm{H} \& \mathrm{E}$ staining of tumor and matched adjacent nontumor tissue containing regions of samples from 3 representative early-stage lung cancer patients. (B) Flow cytometric analysis of lymphocytes from tumor (blue dots) and adjacent (red dots) tissue from early-stage human lung cancer patients. $n=9$ per group. (C) Mean fluorescence intensity of PD-1 in CD4+ and CD8 ${ }^{+}$T cells in tumor (blue dots) and adjacent (red dots) tissue. $n=9$ per group. (D) Representative immunofluorescence staining of tumor and adjacent tissue from early-stage lung cancer patients for CD45 (green), PD-L1 (red), EpCAM (white), and DAPI (blue). Tumor regions are labeled with $\mathrm{T}$; adjacent tissue is labeled with $A$. Staining was performed on 8 samples. Scale bar: $20 \mu \mathrm{m}$. Magnifications for histological and immunohistochemical stains and scale bars for immunofluorescence stains are shown on the images themselves. Two-tailed unpaired $t$ tests with Holm-Sidak correction for multiple comparisons; ${ }^{*} P<0.05,{ }^{* *} P<0.01$.

\section{Tumor}

B Adjacent

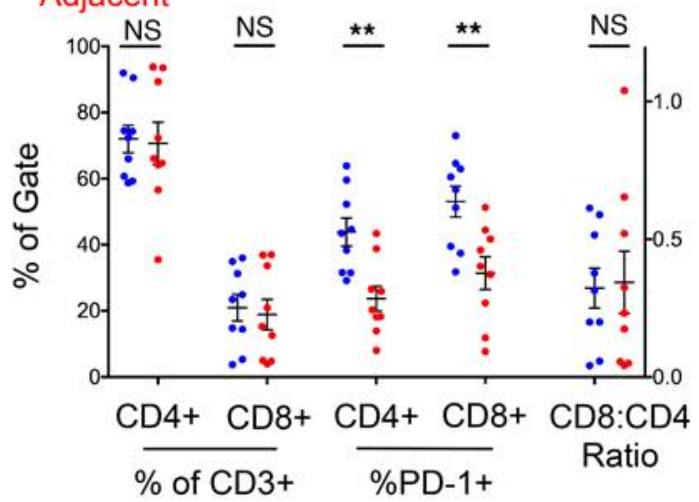

D
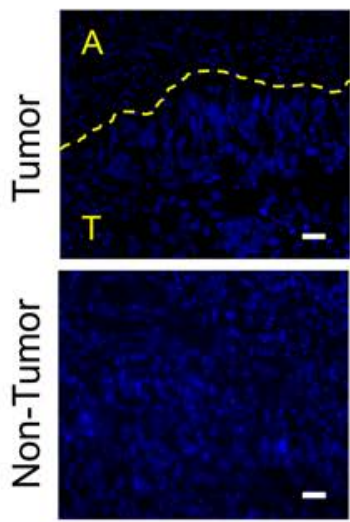

CD45
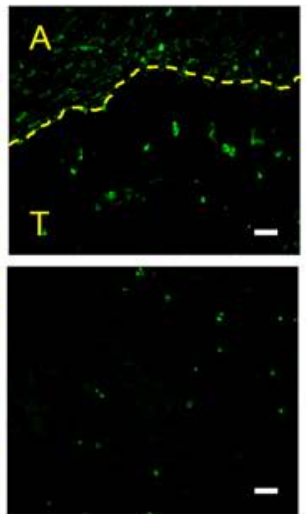

PD-L1
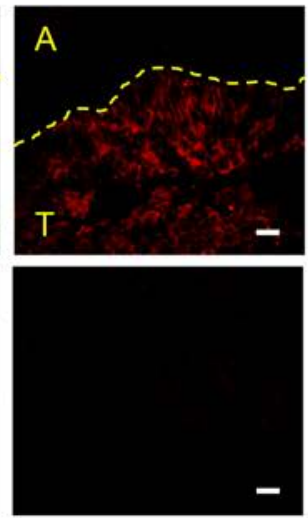

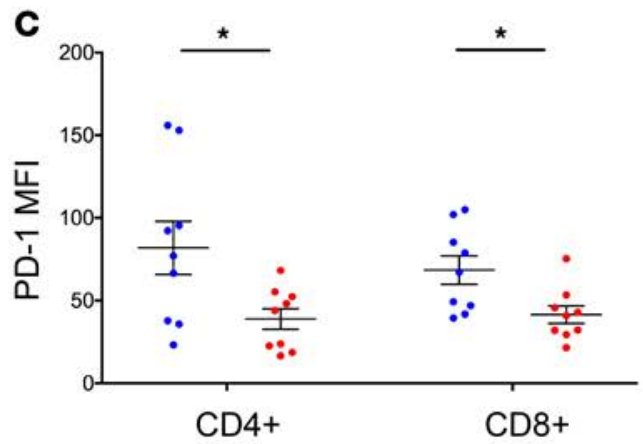

EpCAM
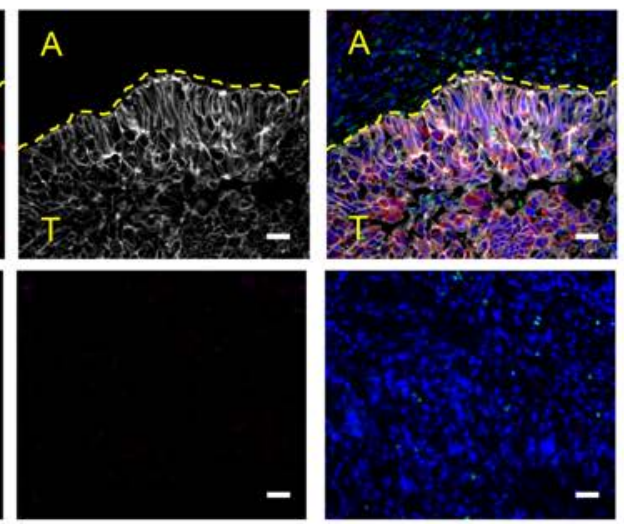
PD-L1 was frequently expressed on the surface of tumor cells and CD45 $5^{+}$leukocytes, indicating potential activation of this immune-suppressive PD-1/PD-L1 axis (Figure 2D). Together, these data suggest that prevalence of the PD-1/PD-L1 axis is conserved in human and murine early-stage NSCLC and provide a rationale for utilizing the HKP1 model for determining the therapeutic efficacy of PD-1 inhibition.

HKP1 tumors develop an immunosuppressive microenvironment as a function of growth. We further analyzed the evolving activity of the immune microenvironment during tumor progression in the HKP1 model. Initially, while $\mathrm{T}$ cells were present in the tumor bed (Supplemental Figure 3A), the $\mathrm{T}$ cell response to the tumor was limited, with low $\mathrm{CD}^{+}$to $\mathrm{CD}^{+} \mathrm{T}$ cell ratios and restrained $\mathrm{T}$ cell proliferation, particularly in $\mathrm{CD}^{+} \mathrm{T}$ cells compared with other lymphocyte subsets (Figure 3, A and B). Effector cytokines, such as IFN- $\gamma$ and TNF- $\alpha$, were not abundant in either $\mathrm{CD} 4^{+}$or $\mathrm{CD} 8^{+} \mathrm{T}$ cells (Figure $3, \mathrm{C}-\mathrm{F}$ ). However, within a short period of tumor growth ( 2 weeks), a robust antitumor $\mathrm{T}$ cell response began to emerge. $\mathrm{T}$ cells began to accumulate robustly in the lungs (Figure $3 \mathrm{~A}$ ). The ratio of $\mathrm{CD} 8^{+}$to $\mathrm{CD} 4^{+} \mathrm{T}$ cells began to rise, with a corresponding marked increase in the proliferation of $\mathrm{CD}^{+} \mathrm{T}$ cells compared with the relatively stable proliferation rate of the other $\mathrm{T}$ cell subsets (Figure 3, A and B). Importantly, effector cytokines, particularly IFN- $\gamma$, were increased in both $\mathrm{CD}^{+}$and $\mathrm{CD} 8^{+} \mathrm{T}$ cells (Figure $3, \mathrm{C}-\mathrm{F}$ ). Increased $\mathrm{T}$ cell proliferation, $\mathrm{CD} 8^{+}$ to $\mathrm{CD}^{+} \mathrm{T}$ cell ratio, and effector cytokine production suggest an ongoing active antitumor immunity.

In contrast, evaluation of tumors at a later time point (3 weeks) indicated a progressively dysfunctional immune response. While T cells continued to accumulate in the lung, their infiltration of the tumor beds became restricted primarily to the tumor margin (Supplemental Figure 3A). Tumors also showed hallmarks of immunosuppression, as demonstrated by increased accumulation of $\mathrm{CD}^{+} \mathrm{FoxP} 3^{+}$Tregs (Figure $3 \mathrm{~A}$ ) and decreased effector cytokine production, particularly in the $\mathrm{CD}^{+} \mathrm{T}$ cell pool (Figure 3, C-F), demonstrating diminished effector capacity. Notably, this was associated with increased expression of PD-1 on all lymphocyte subsets, including $\mathrm{CD} 8^{+} \mathrm{T}$ cells, Tregs, and $\mathrm{CD} 4^{+} \mathrm{FoxP} 3^{-} \mathrm{Th}$ cells, indicative of global $\mathrm{T}$ cell dysfunction (Supplemental Figure 4). Further checkpoint proteins, including 2B4 and LAG-3, also began to differentially increase in $\mathrm{T}$ cell subsets late in disease progression, while Eomes expression decreased and T-Bet expression increased at the latest stages in $\mathrm{CD}^{+} \mathrm{T}$ cells (Supplemental Figures 5 and 6). We further analyzed the myeloid compartment, given that these cells contribute significantly to the immune microenvironment and are a determinant of response to checkpoint blockade $(24,26,27)$. Our analysis showed an increase in $\mathrm{CD} 11 \mathrm{~b}^{+} \mathrm{CD} 11 \mathrm{c}^{-} \mathrm{Gr} 1^{\text {hi }}$ neutrophils/polymorphonuclear myeloid-derived suppressor cells (PMN-MDSCs) and corresponding decreases in $\mathrm{CD} 11 \mathrm{~b}^{+} \mathrm{CD} 11 \mathrm{c}^{-} \mathrm{Gr} 1^{\mathrm{mid}}$ monocytic myeloid-derived suppressor cells (Mo-MDSCs) and $\mathrm{CD} 11 \mathrm{~b}^{+} \mathrm{CD} 11 \mathrm{c}^{-} \mathrm{Gr} 1^{\text {lo }}$ mature macrophages as tumors progressed (Supplemental Figure 7A). Functional analysis of these populations demonstrated increased NOS2 expression over time in $\mathrm{CD}_{11 \mathrm{~b}^{+}}$myeloid subsets, while indoleamine 2,3-dioxygenase (IDO) production was temporally regulated and more restricted to Mo-MDSCs (Supplemental Figure 7, B and C).

Taken together, these data suggest that the robust antitumor immune response in the HKP1 model progressively converts to an immunosuppressive phenotype, as observed in NSCLC patients. This finding provided the rationale to test the therapeutic efficacy and mechanistic effects of PD-1 inhibition in early-stage NSCLC.

Anti-PD-1 treatment results in tumor control and improved overall survival by altering the immune microenvironment. To determine the therapeutic efficacy of PD-1 inhibition in early-stage NSCLC, we generated cohorts of HKP1 tumor-bearing mice and administered clinically relevant doses of a rat monoclonal blocking anti-PD-1 antibody (clone RMP1-14; 4 doses of $250 \mu \mathrm{g}$ ) over a 2-week period starting 1 week following tumor implantation. We observed a significant survival benefit: median survival was 22 days in control IgG2a-treated mice (clone 2A3) compared with 29.5 days in anti-PD-1-treated mice (Figure 4A, $P$ $=0.0037)$. Evaluation of tumor growth by bioluminescence imaging showed overall slower tumor growth in the cohort of mice treated with anti-PD-1 (Figure 4B). Interestingly, there was variability in the degree of response to anti-PD-1, with a subset of mice displaying similarly rapid growth kinetics as those observed in mice treated with IgG2a (Supplemental Figure 8). As expected, anti-PD-1 treatment was associated with a robust $\mathrm{T}$ cell infiltrate in tumor beds (Figure $4 \mathrm{C}$ ).

To better understand the impact of single-agent anti-PD-1 on increased survival and impaired tumor growth in early-stage NSCLC, we examined the tumor immune microenvironment following the last administration of the drug. Accumulation of $\mathrm{CD}^{+} \mathrm{T}$ lymphocytes in tumor-bearing lungs of mice treated with antiPD-1 antibody was significantly enhanced compared with that in controls (Figure 5A). Interestingly, anti-PD-1 treatment robustly increased proliferation of all lymphocyte subsets, including $\mathrm{CD} 8^{+} \mathrm{T}$ cells, Tregs, and $\mathrm{Th}$ cells, irrespective of their accumulation state (Figure 5B). This also corresponded with significantly increased 
A
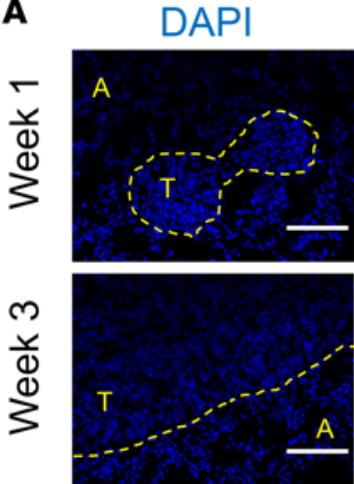

E-Cadherin
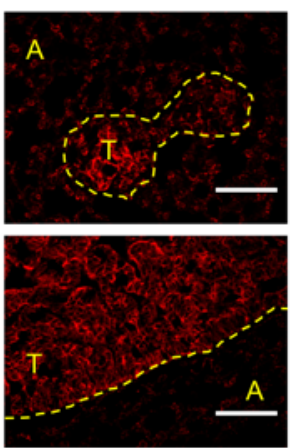

CD3
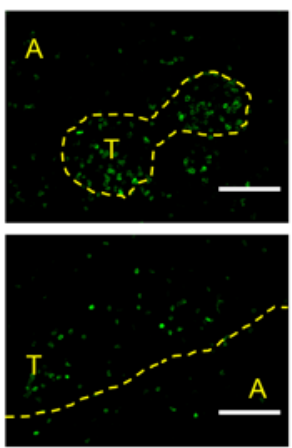

Merged
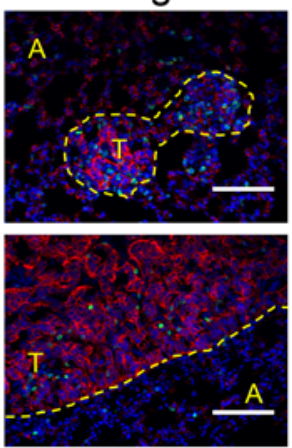

$H \& E$

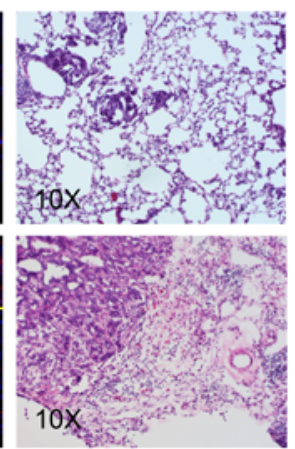

B

Week 1

Week 2

Week 3

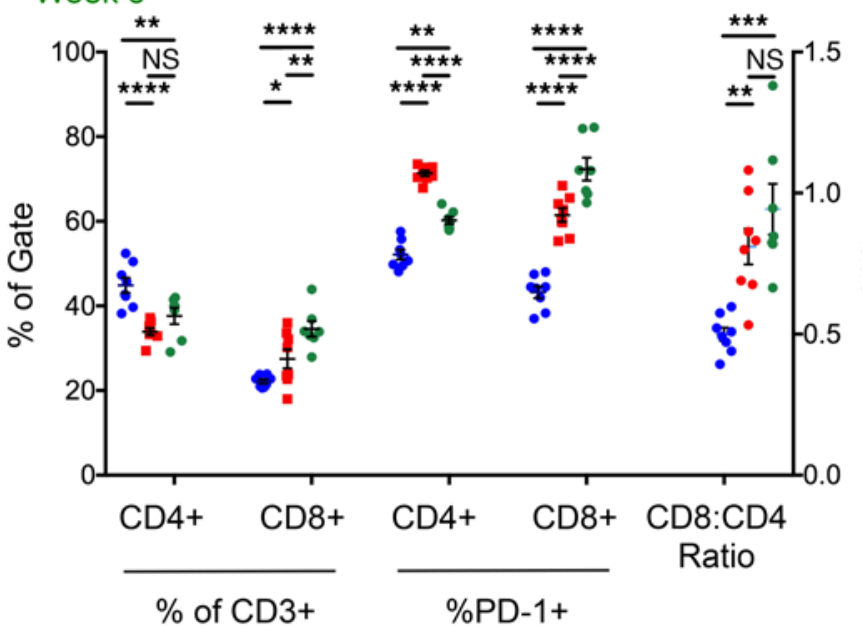

D
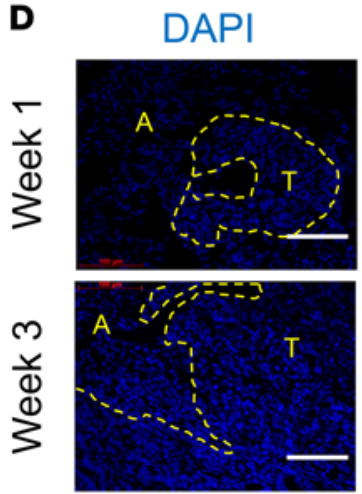

\section{CD45}
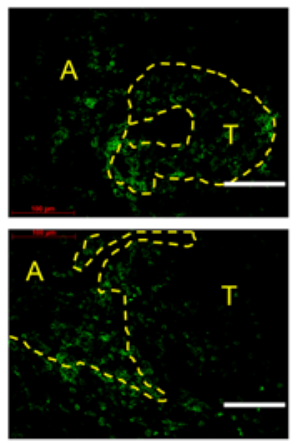

C

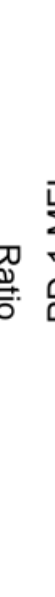

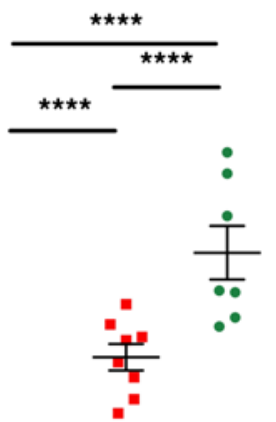

$\stackrel{\bullet=0}{\rightarrow=0}$

CD8+
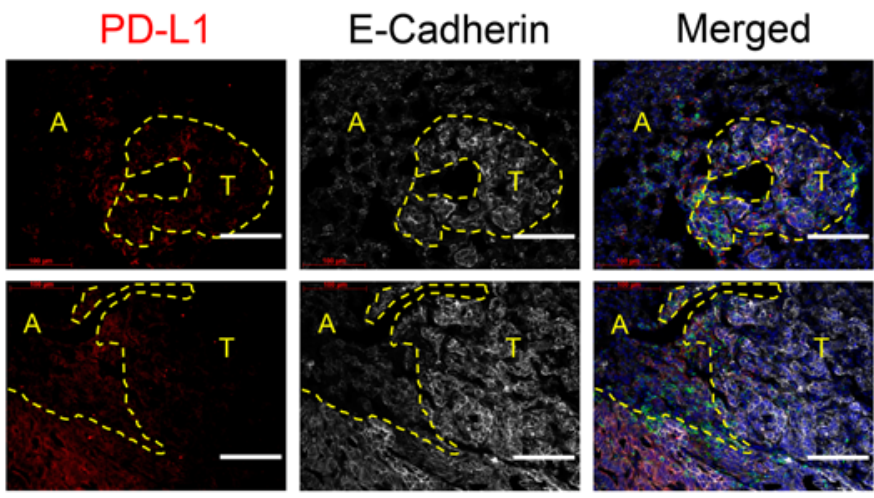

Figure 2. Tumor-infiltrating lymphocyte accumulation and PD-1/PD-L1 axis as a function of tumor progression in HKP1 orthotopic model of lung cancer. (A) Representative immunofluorescence for E-cadherin (red), CD3 (green), and DAPI (blue), and H\&E staining of tumor-bearing lung tissue 1 week and 3 weeks after implantation. Tumor regions are labeled with T; adjacent tissue is labeled with A. Staining was performed on 3 samples. (B) Flow cytometric analysis of lymphocytes from lungs 1 week (blue dots), 2 weeks (red dots), and 3 weeks (green dots) after implantation. $n=7-8$ per group. (C) Mean fluorescence intensity of PD-1 in CD4 ${ }^{+}$and CD8 ${ }^{+}$T cells from lungs 1 week (blue dots), 2 weeks (red dots), and 3 weeks (green dots) after implantation. $n=$ 7-8 per group. (D) Representative immunofluorescence staining of tumor-bearing lung tissue 1 week and 3 weeks for CD45 (green), PD-L1 (red), E-cadherin (white), and DAPI (blue). Tumor regions are labeled with T; adjacent tissue is labeled with A. Staining was performed on 3 samples. Scale bar: $100 \mu$ m. Magnifications for histological and immunohistochemical stains and scale bars for immunofluorescence stains are shown on the images themselves. Oneway ANOVA with Tukey's multiple comparisons test for ratios, 2-way ANOVAs with Tukey's multiple comparisons test for all other analyses; ${ }^{*} P<0.05$, ${ }^{* *} P$ $<0.01,{ }^{* *} P<0.001,{ }^{* * *} P<0.0001$. 
Week 1

A Week 2

Week 3

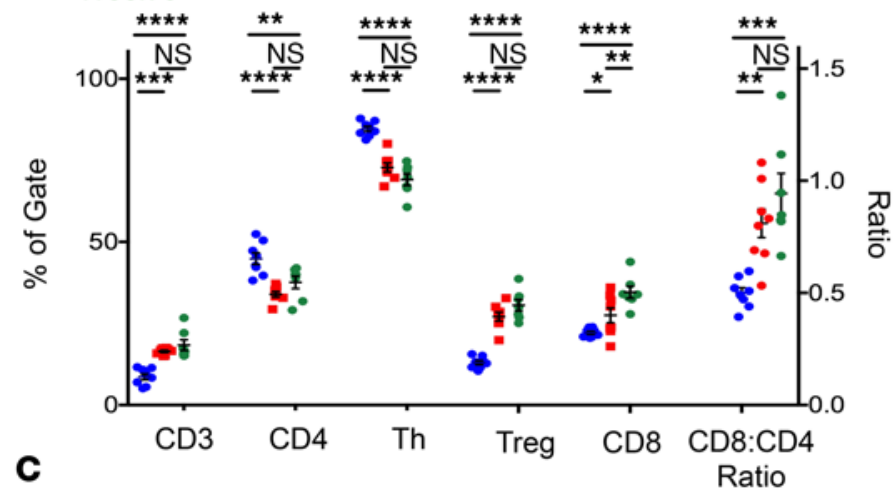

D
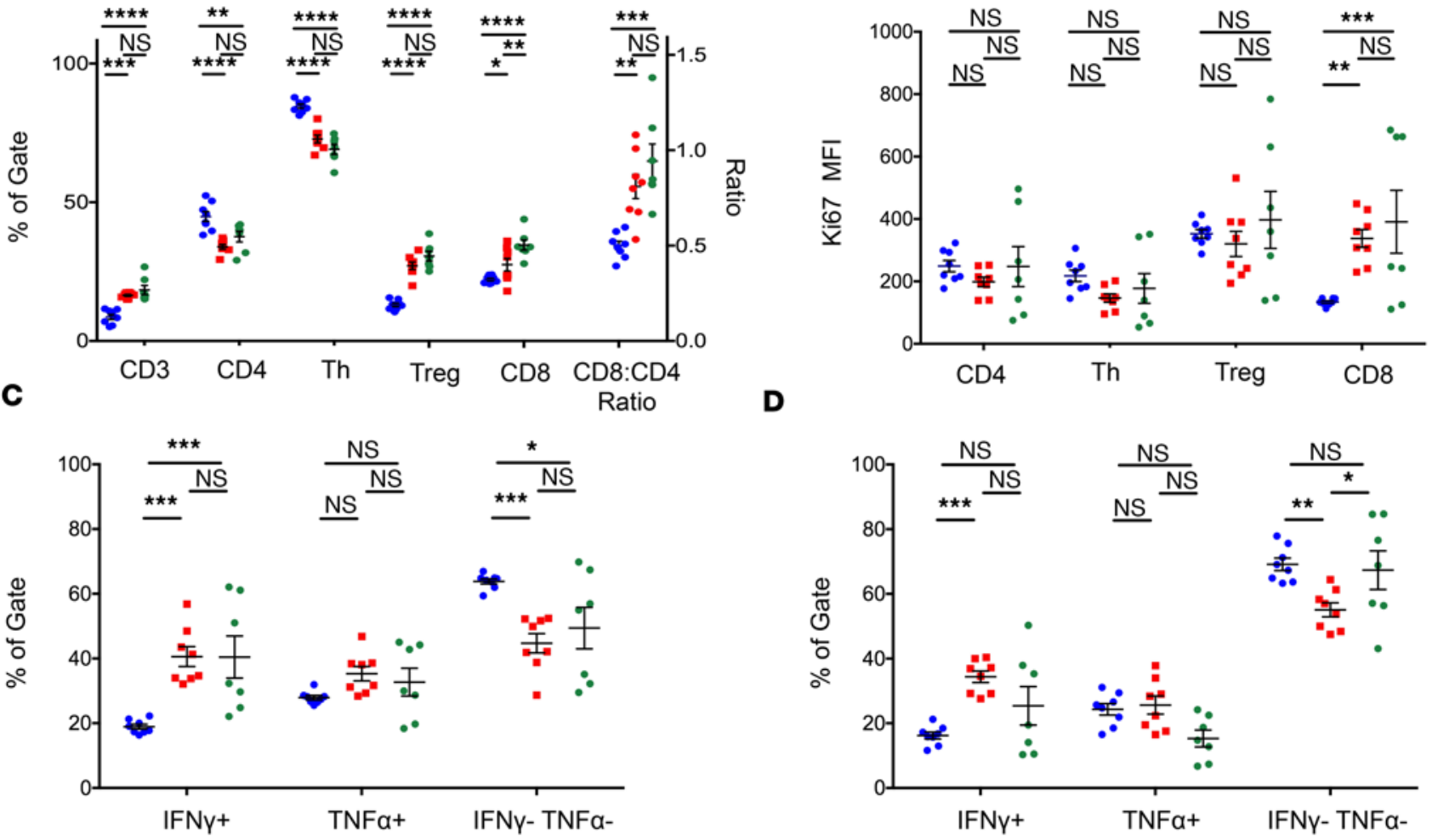

E

CD4

$\mathbf{F}$

CD8

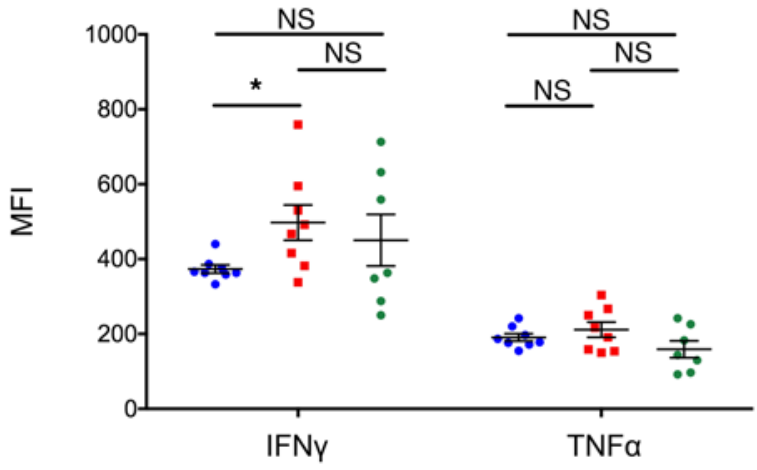

CD4

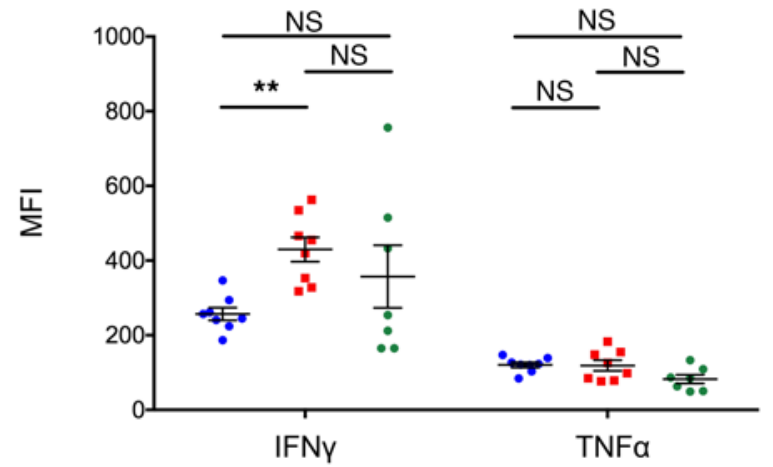

CD8

Figure 3. Differential T cell populations, proliferation, and cytokine production as a function of tumor progression in the HKP1 model of lung cancer. Flow cytometric analysis of tumor-bearing lung tissue 1 week (blue dots), 2 weeks (red dots), and 3 weeks (green dots) after tumor implantation

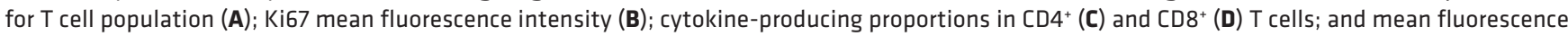
intensity of IFN $-\gamma$ and TNF- $\alpha$ in CD4 ${ }^{+}$(E) and CD8 ${ }^{+}$(F) T cells. $n=7-8$ per group. Two-way ANOVAs with Tukey's multiple comparisons test; ${ }^{*} P<0.05$, ${ }^{* *} P<0.01,{ }^{* * *} P<0.001,{ }^{* * *} P<0.0001$.

surface PD-1 expression on CD4 ${ }^{+} \mathrm{T}$ cells and Th cells, while PD-1 expression on $\mathrm{CD}^{+} \mathrm{T}$ cells was unchanged as a result of PD-1 inhibition (Supplemental Figure 9A). Strikingly, surface PD-1 expression increased on Tregs, leading to a significantly larger pool of Tregs that were PD- $1^{+}$in the anti-PD-1-treated mice compared with controls (Supplemental Figure 9, A and B). Next, we evaluated the effect of PD-1 inhibition on effector functions of $\mathrm{T}$ cells. We observed a trend toward an increase in the proportion of $\mathrm{CD} 4^{+} \mathrm{T}$ cells expressing IFN- $\gamma$ or TNF- $\alpha$ (Figure 5C); more importantly, this corresponded with a higher mean expression of effector 
A

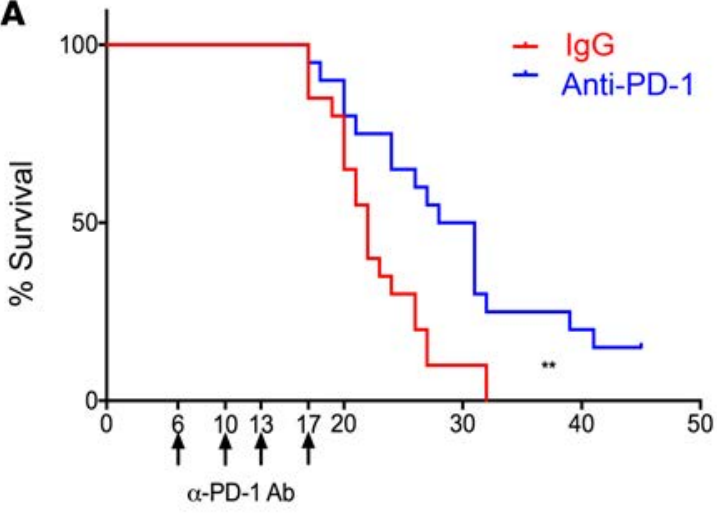

Tumor growth (days)
B

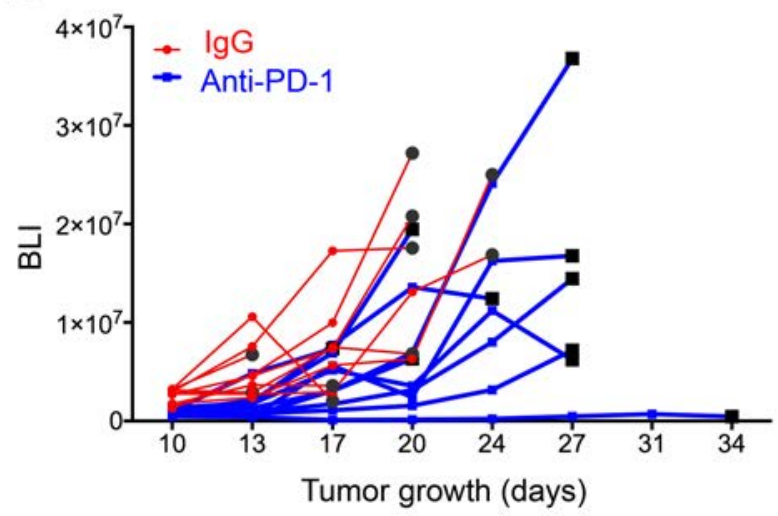

C
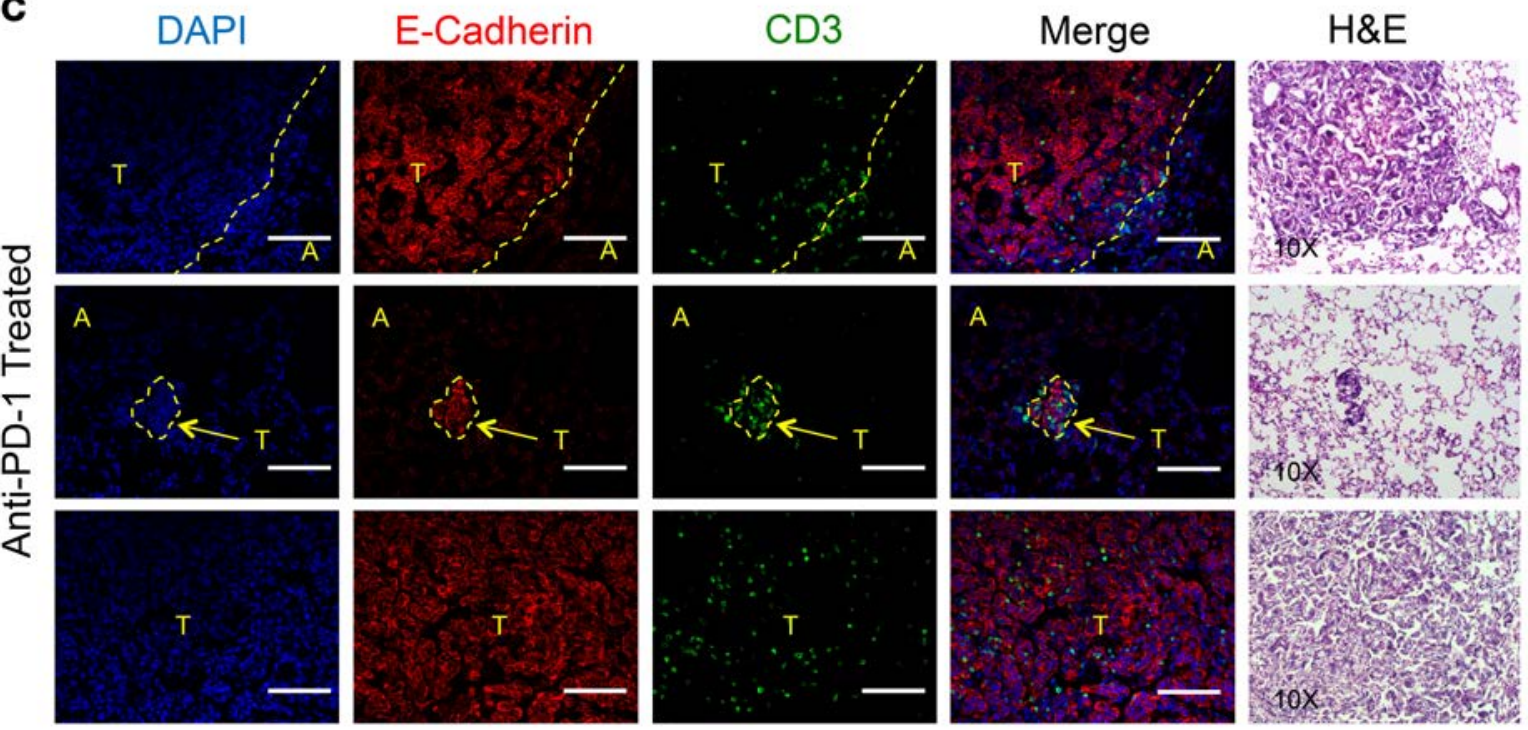

Figure 4. Anti-PD-1 therapy initiated early in tumor progression results in significant survival benefits in HKP1 lung cancer model. (A) Kaplan-Meier survival curve of mice treated with $250 \mu \mathrm{g}$ per dose of either IgG2a (red line) or anti-PD-1 (blue line) on days 6, 10, 13, and 17 after implantation. $n=20$ per group. (B) Bioluminescence imaging (BLI) data measuring tumor growth in IgG2a-treated (red lines) or anti-PD-1-treated (blue lines) mice. Black symbols indicate the last time point at which a given mouse was imaged before euthanasia or mortality. $n=10$ per group (median 10 of total 20 mice). (C) Representative immunofluorescence for E-cadherin (red), CD3 (green), and DAPI (blue) and H\&E staining of 3 fields of view of tumor-bearing lung tissue from mice treated with anti-PD-1 and harvested 18 days after implantation, 1 day after the last dose of anti-PD-1. Tumor regions are labeled with T; adjacent tissue is labeled with A. Staining was performed on 3 samples. Scale bar: $100 \mu \mathrm{m}$. Magnifications for histological and immunohistochemical stains and scale bars for immunofluorescence stains are shown on the images themselves. Log-rank (Mantel-Cox) test for survival; ${ }^{* *} P<0.01$.

cytokines IFN- $\gamma$ and TNF- $\alpha$ in $\mathrm{CD} 4^{+} \mathrm{T}$ cells in mice treated with anti-PD-1 compared with controls (Figure $5 \mathrm{E}$ ), although no significant changes in GzmB, IL-2, or FasL were observed (Supplemental Figure 10A). PD-1+ Tregs, which accumulated in anti-PD-1-treated mice, also produced more IFN- $\gamma$ than their PD-1- counterparts (Supplemental Figure 9C). Surprisingly, PD-1 inhibition did not increase expression of either IFN- $\gamma$ or TNF- $\alpha$ in $\mathrm{CD}^{+} \mathrm{T}$ cells (Figure 5, D and F). Similarly, there was no increase in the expression of GzmB, IL-2, or FasL in $\mathrm{CD} 8^{+} \mathrm{T}$ cells (Supplemental Figure 10B). Further analyses showed that PD-1 inhibition did not affect generation of effector or effector memory cells in either $\mathrm{CD} 4^{+}$or $\mathrm{CD} 8^{+} \mathrm{T}$ cells (Supplemental Figure 10C). In the myeloid compartment, PD-1 inhibition significantly decreased the accumulation of $\mathrm{CD} 11 \mathrm{~b}^{+} \mathrm{CD} 11 \mathrm{c}^{+}$cells; in the $\mathrm{CD} 11 \mathrm{~b}^{+} \mathrm{CD} 11 \mathrm{c}^{-}$subset, neutrophils/PMN-MDSCs were proportionally significantly reduced, while a significant increase in the proportion of mature macrophages was observed (Supplemental Figure 10D). Functional analysis of the myeloid populations demonstrated that anti-PD-1 treatment increased NOS2 expression in all myeloid subsets, while IDO production was enriched in Mo-MDSCs and reduced in mature macrophages and $\mathrm{CD} 11 \mathrm{~b}^{-} \mathrm{CD} 11 \mathrm{c}^{+}$dendritic cells (Supplemental Figure 10, E and F). 
$\lg G$

A Anti-PD-1

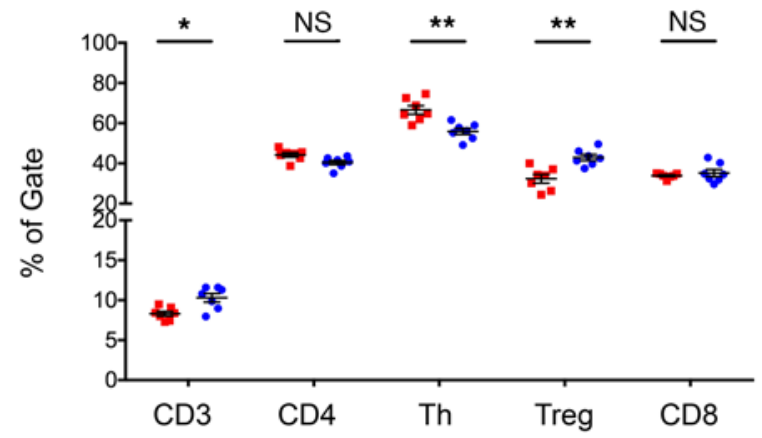

C

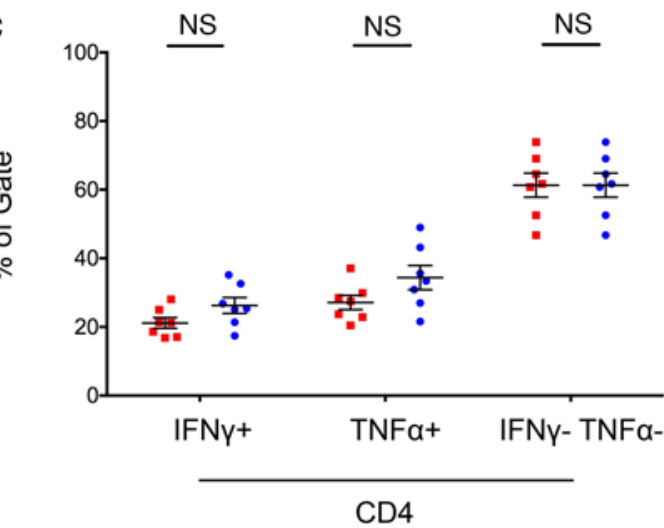

$\mathbf{E}$

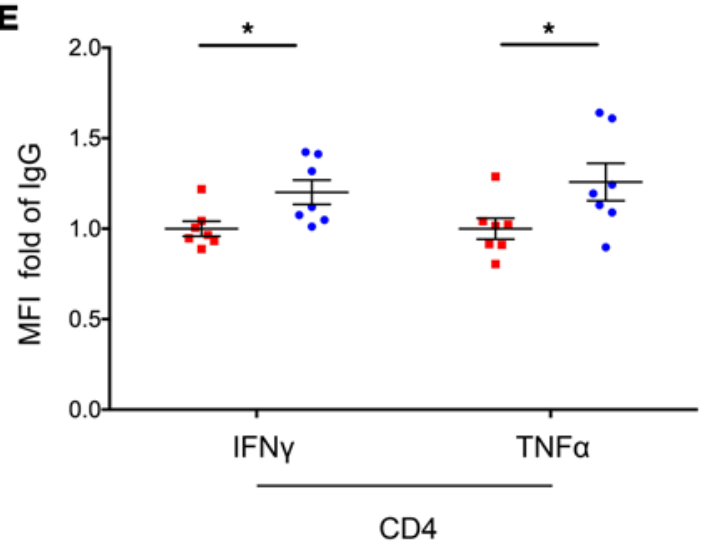

B

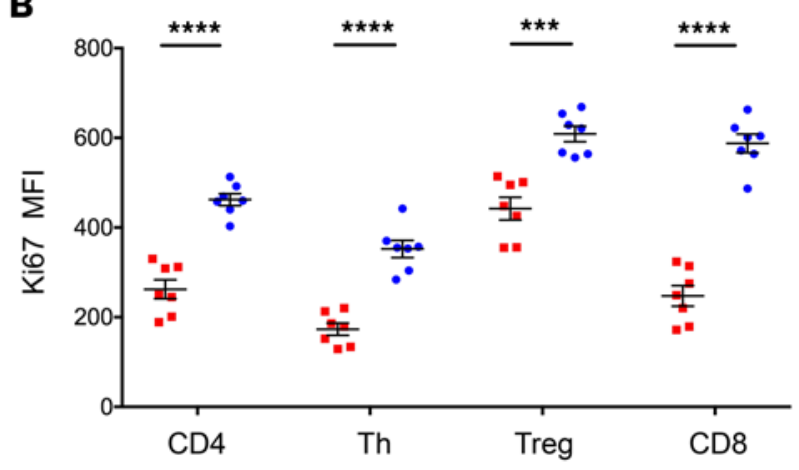

D

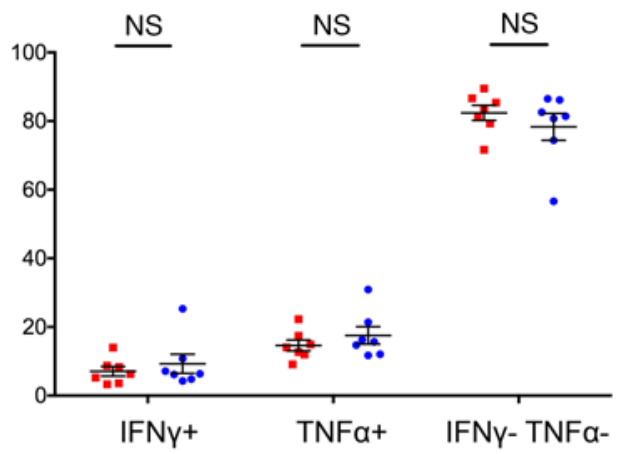

CD8

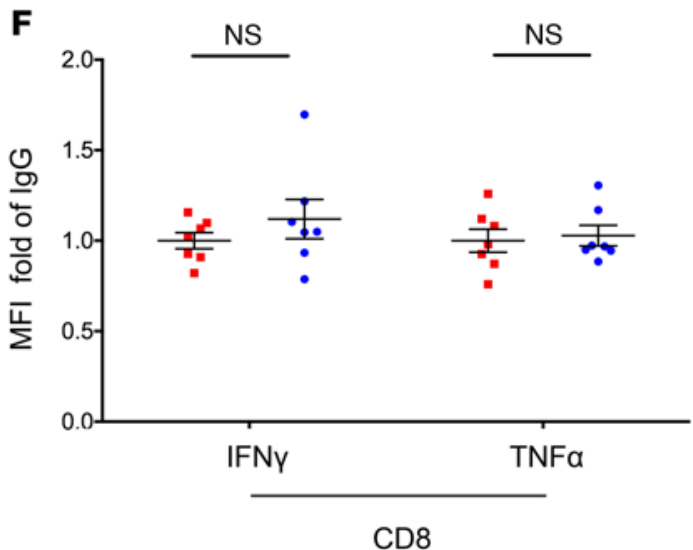

Figure 5. Differential T cell populations, proliferation, and cytokine production upon anti-PD-1 treatment in HKP1 lung cancer model. Flow cytometric analysis of tumor-bearing lung tissue from mice treated with IgG2a (red dots) or anti-PD-1 (blue dots) for T cell population proportions (A); Ki67 mean fluorescence intensity (B); cytokine-producing proportions of $\mathrm{CD4}^{+}(\mathbf{C})$ and $\mathrm{CD}^{+}(\mathbf{D})$ T cells; and mean fluorescence intensity of IFN- $\gamma$ and TNF- $\alpha$ in anti-PD-1treated $\mathrm{CD}^{+}(\mathbf{E})$ and $\mathrm{CD}^{+}(\mathbf{F})$ T cells normalized to the mean fluorescence intensity of the same in lgG2a-treated mice. $n=7$ per group. Two-tailed unpaired $t$ tests with Holm-Sidak correction for multiple comparisons. ${ }^{*} P<0.05,{ }^{* *} P<0.01,{ }^{* *} P<0.001,{ }^{* * * *} P<0.0001$.

Taken together, these data suggest that the efficacy of PD-1 inhibition in early tumors may be attributed to increased $\mathrm{T}$ cell abundance, proliferation, and effector cytokine production and a concomitant decrease in immunosuppressive myeloid cells.

Depletion of both $C D 4^{+}$and $C D 8^{+} T$ cell subsets results in diminished efficacy of anti-PD-1, with synergistic effects. We have demonstrated that PD-1 inhibition results in significant tumor control and improved overall survival associated with an enhancement in $\mathrm{CD}^{+}$and $\mathrm{CD}^{+} \mathrm{T}$ cell proliferation and increased effector cytokine production in $\mathrm{CD}^{+} \mathrm{T}$ cells. To directly demonstrate that these $\mathrm{T}$ cell populations drive the survival benefit of anti-PD-1 therapy in the HKP1 tumor model, we sought to specifically deplete $\mathrm{CD} 4^{+}$and $\mathrm{CD} 8^{+} \mathrm{T}$ cells to determine their effect on the efficacy of PD-1 inhibition. We generated cohorts of HKP1 tumor-bearing 
mice and administered anti-PD-1 antibody alone or in combination with depleting antibodies targeting CD $4^{+}$ $\mathrm{T}$ cells (clone GK1.5), $\mathrm{CD}^{+} \mathrm{T}$ cells (clone 2.43 ), both $\mathrm{CD}^{+}$and $\mathrm{CD} 8^{+} \mathrm{T}$ cells, and controls (IgG2b clone LTF-2) (Figure 6A). Submandibular bleeds 1 day after the first and last dose of depleting antibody (days 7 and 16, respectively; Supplemental Figure 11) confirmed significant depletion of targeted subsets $(P<0.0001$ vs. anti-PD-1 + IgG for all comparisons), with the exception of one mouse treated with anti-CD4, which did not initially show substantial depletion at day 7; however, robust depletion was also achieved in that mouse by day 16 (Figure 6B). Tumor growth monitored by bioluminescence imaging revealed that both $\mathrm{CD}^{+}$and $\mathrm{CD}^{+} \mathrm{T}$ cell depletion resulted in enhanced growth in anti-PD-1 treated mice compared with nondepleted controls, indicating that both populations contribute to anti-PD-1's efficacy in controlling tumor progression (Figure 6C and Supplemental Figure 12, A-C). As expected, depletion of CD8 ${ }^{+} \mathrm{T}$ cells had a larger effect on anti-PD-1-mediated tumor control, given their canonical function to directly kill tumor cells, while CD4 ${ }^{+} \mathrm{T}$ cell depletion had a more moderate and delayed effect on anti-PD-1-mediated tumor control (Figure 6C). While depletion of $\mathrm{CD}^{+} \mathrm{T}$ cells alone accelerated tumor growth in the majority of samples, a subset of tumors demonstrated substantial anti-PD-1-mediated tumor control (Supplemental Figure 12C). Interestingly, the single mouse that displayed delayed $\mathrm{CD}^{+} \mathrm{T}$ cell depletion demonstrated enhanced tumor progression compared with nondepleted controls, yet reduced efficacy compared with the majority of the other CD4 $4^{+} \mathrm{T}$ cell-depleted tumors (Supplemental Figure 12C, red arrow). Importantly, depletion of both CD4 ${ }^{+}$and CD8 ${ }^{+}$ $\mathrm{T}$ cells resulted in a synergistic effect, with greatly enhanced tumor growth in the context of anti-PD-1 treatment (Figure 6C and Supplemental Figure 12D).

These data demonstrate that both $\mathrm{CD}^{+}$and $\mathrm{CD} 8^{+} \mathrm{T}$ cells play critical roles in inducing anti-PD-1-mediated tumor control, with their significant synergistic effects suggesting a robust interplay between the two populations to control tumor progression.

Differential reprogramming of transcriptome of tumor-infiltrating $T$ cells upon treatment with anti-PD-1. Our data demonstrated that treatment with anti-PD-1 in early murine NSCLC resulted in significant alterations in T cell abundance, proliferation, and effector cytokine production. However, these effects were not consistent between $\mathrm{CD}^{+}$and $\mathrm{CD} 8^{+} \mathrm{T}$ cells, suggesting a differential response to anti-PD-1 therapy between the $\mathrm{T}$ lymphocyte subsets. We therefore examined the transcriptional landscape of bulk $\mathrm{CD} 4^{+}$and $\mathrm{CD}^{+} \mathrm{T}$ cells isolated from tumor-bearing lungs at multiple time points after treatment with anti-PD-1 or IgG2a using RNA sequencing. Consistent with previous results, treatment with anti-PD-1 resulted in decreased tumor burden compared with treatment with IgG2a control (Figure 7A). As expected, there was variability in the magnitude of response to anti-PD-1 treatment, with the majority of samples demonstrating substantial remission, while a subset demonstrated limited effects on tumor progression (Figure 7A). Principle component analysis (PCA) of transcriptomic data of $\mathrm{CD}^{+}$and $\mathrm{CD} 8^{+} \mathrm{T}$ cells isolated from lungs demonstrated clustering that corresponded to these observed responses to anti-PD-1 treatment (Figure 7B).

We subsequently interrogated differences in gene expression in $\mathrm{CD}^{+}$and $\mathrm{CD} 8^{+} \mathrm{T}$ cells. A panel of genes associated with exhaustion (28), including transcriptional regulators (such as Eomes, Tbx21, Ikzf2, Id2, and Litaf), coinhibitory and costimulatory molecules (such as Pdcd1, Ctla4, Havcr2, Tigit, Cd80, and Cd86), and cytokines and chemokines and their cognate receptors (such as Ifng, Tnf, Il12a, Gzmb, Il2ra, and Ifngr2), segregated the samples into 6 distinct groups matching tumor growth and principle component analysis analyses: IgG2a-treated progressing tumors (group 1), early anti-PD-1-treated regressing tumors (group 2), early antiPD-1-treated progressing tumors (group 3), late anti-PD-1-treated regressing tumors (group 4), late antiPD-1-treated partially regressing tumors (group 5), and late anti-PD-1-treated progressing tumors (group 6) (Figure 8 and Supplemental Figure 13). Samples from mice treated with anti-PD-1 in which the tumor still demonstrated growth at day 14 (group 3) and at later time points (days 17 and 24, group 6) clustered more closely with samples from mice treated with IgG2a (group 1; Figure 7, A and B, and Figure 8), mirroring their similar in vivo growth patterns. Samples from mice treated with anti-PD-1 in which the tumor demonstrated significant tumor regression at day 14 (group 2) and at later time points (days 17 and 24; group 4) showed distinct gene expression profiles from groups 1, 3, and 6, while samples from mice treated with anti-PD-1 in which the tumor demonstrated an intermediate growth inhibition phenotype at later time points (days 17 and 24; group 5) exhibited a more heterogeneous gene expression profile (Figure 7, A and B, and Figure 8).

We further investigated prevalence of $\mathrm{CD}^{+} \mathrm{T}$ cell subsets by analyzing transcription factor expression (Supplemental Figure 14) (28). We observed time-, treatment-, and tumor burden-dependent effects on $\mathrm{CD}^{+}$subset abundance. Treg abundance, demonstrated by Foxp3 expression, shifted dramatically between groups, becoming highly prevalent in the groups with larger tumor burdens (groups 1, 3, and 6) and most 
A

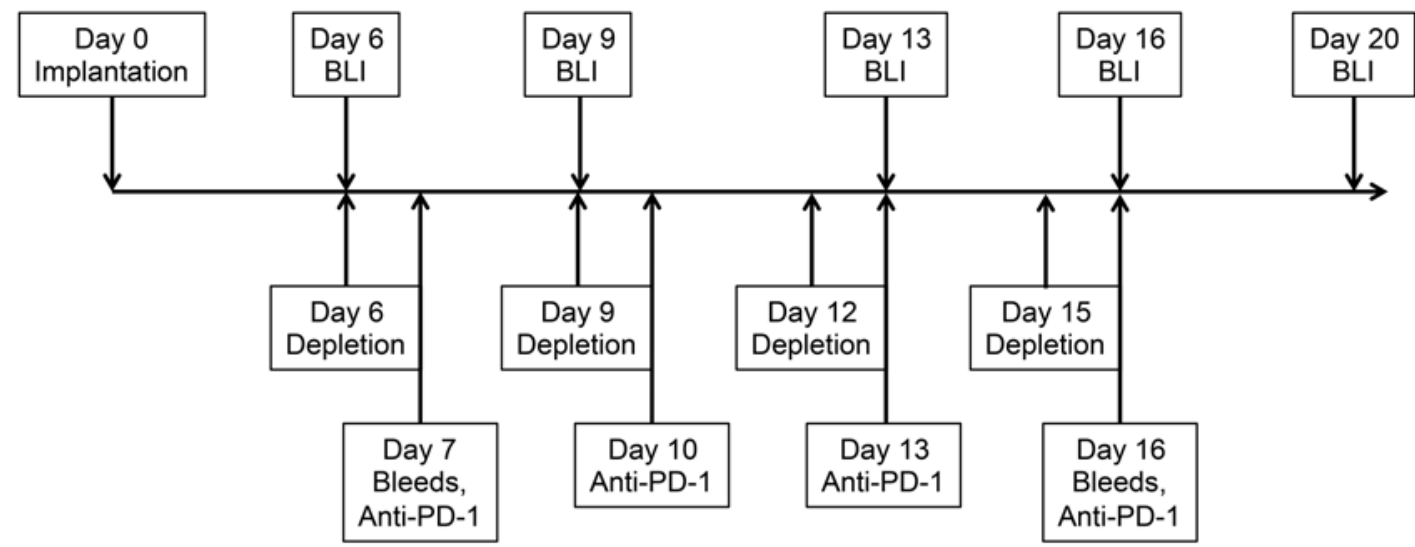

B

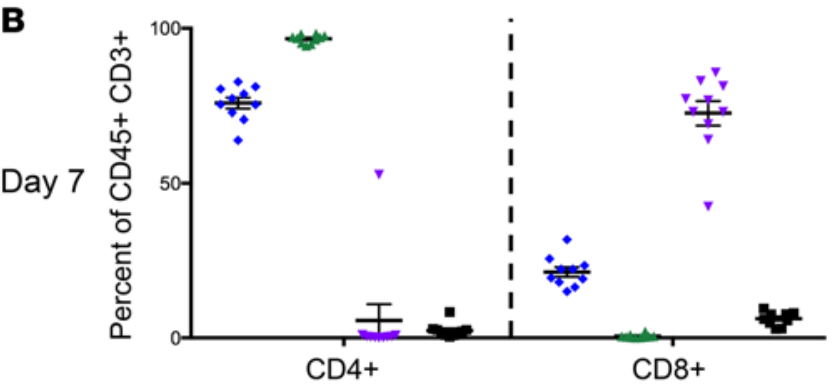

C

Anti-PD-1 + IgG

Anti-PD-1 + anti-CD8a

Anti-PD-1 + anti-CD4

Anti-PD-1 + anti-CD4 + anti-CD8 $\alpha$
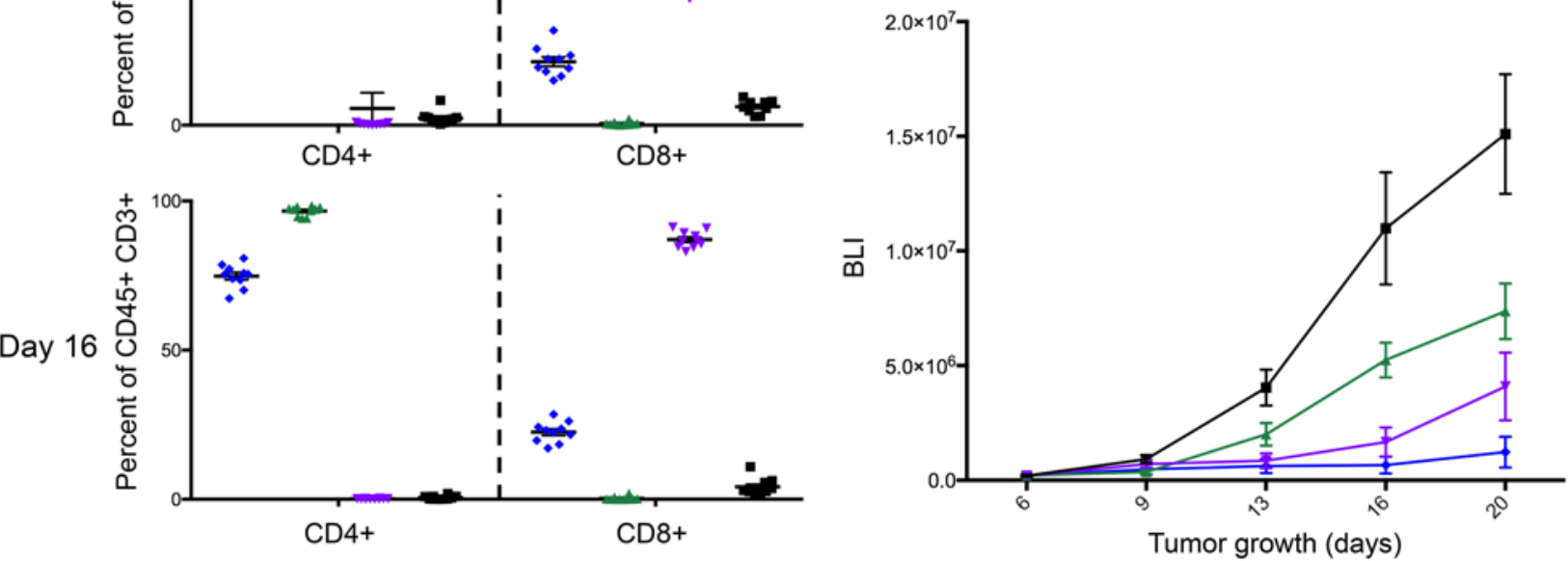

Figure 6. Depletion of T cell subsets synergistically affects anti-PD-1 therapeutic efficacy. (A) Experimental schematic. (B) Flow cytometric analysis of peripheral blood for $\mathrm{T}$ cell population proportions on days 7 and 16 after implantation following depletion of no T cell subsets (blue dots); CD8 ${ }^{+} \mathrm{T}$ cells (green dots); $C D 4^{+} T$ cells (purple dots); or $C D 8^{+}$and $C D 4^{+} T$ cells (black dots). (C) Bioluminescence imaging (BLI) data measuring tumor growth in anti-PD-1-treated mice with depletion of no T cell subsets (blue line); $C D 8^{+} T$ cells (green line); $C D 4^{+} T$ cells (purple line); or $C D 8^{+}$and $C D 4^{+} T$ cells (black line). $n=8-10$ per group.

abundant at the latest time point analyzed (group 6). T follicular helper cells, characterized by Bcl6 expression, were enriched in groups with larger tumor burdens at early time points (groups 1 and 3) but were less prevalent at later time points. Th2 cells, expressing Gata3, had a markedly high abundance in IgG-treated mice at early time points (group 1) compared with any of the anti-PD-1-treated cohorts. Similarly, Th17 cells, expressing Rorc, had heterogeneous expression in the IgG-treated group 1 but little expression in any anti-PD-1-treated cohorts. Finally, Th1 cells, expressing Tbx21, were more abundant at initial time points (groups 1,2, and 3) but were dramatically depleted in samples from later time points (groups 4, 5, and 6) and notably even more so in those samples with tumor control (groups 4 and 5).

Having observed significant clustering of our samples, utilizing known regulators of exhaustion and shifts in T cell subpopulations, we explored global differences in gene expression corresponding to these response patterns to anti-PD-1 therapy. We first examined genes differentially regulated in a robust antitumor response to anti-PD-1 therapy, both during treatment (group 2) and at later time points (group 4), compared with IgG2a-treated mice during treatment (group 1). Multiple factors implicated in modulating long-term antigen exposure and exhaustion phenotypes, including coinhibitory molecules, such as $P d c d 1$, Havcr2, and Tigit; costimulatory molecules, such as Cd80,Tnfrsf4, and Tnfrsf9; and transcription regulators, such as $\operatorname{Ikzf2}$, Prdm1, and Batf, had significantly decreased expression (Figure 8). Conversely, progressive 
A

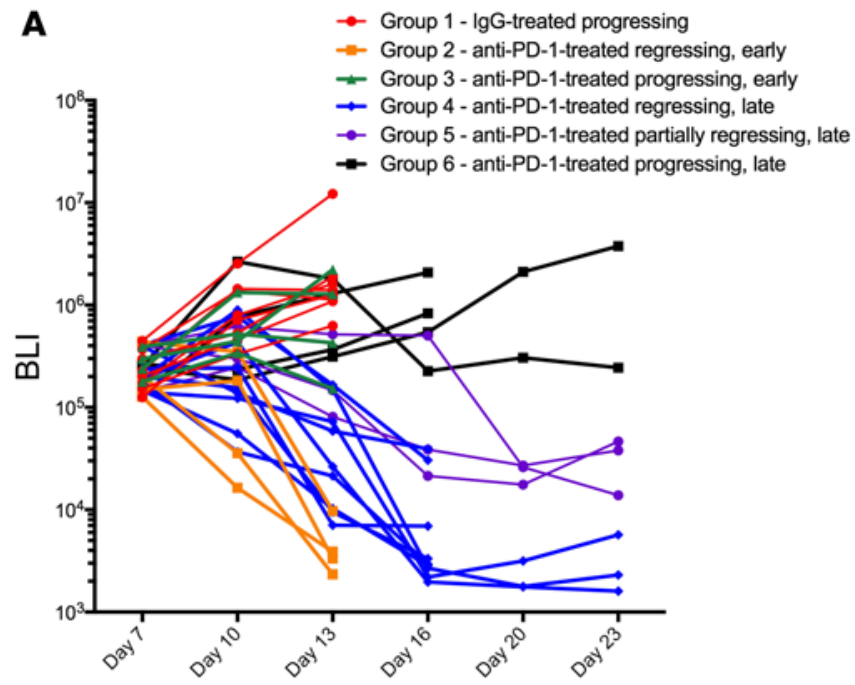

Figure 7. Differential reprogramming of the transcriptome of tumor-infiltrating $T$ cell subsets upon anti-PD-1 treatment in HKP1 lung cancer model. (A) Bioluminescence imaging (BLI) data measuring tumor growth in IgC2a-treated (group 1; red lines) or anti-PD-1-treated (groups 2-6; orange, green, blue, purple, and black lines, respectively) mice. (B) Principle component analysis of sequencing data from $\mathrm{CD}^{+}$(left) and CD8 $8^{+}$(right) T cells isolated from tumor-bearing lung tissue from mice treated with IgC2a (group 1; red dots) or anti-PD-1 (groups 2-6; orange, green, blue, purple, and black dots, respectively).

Tumor growth (days)

B
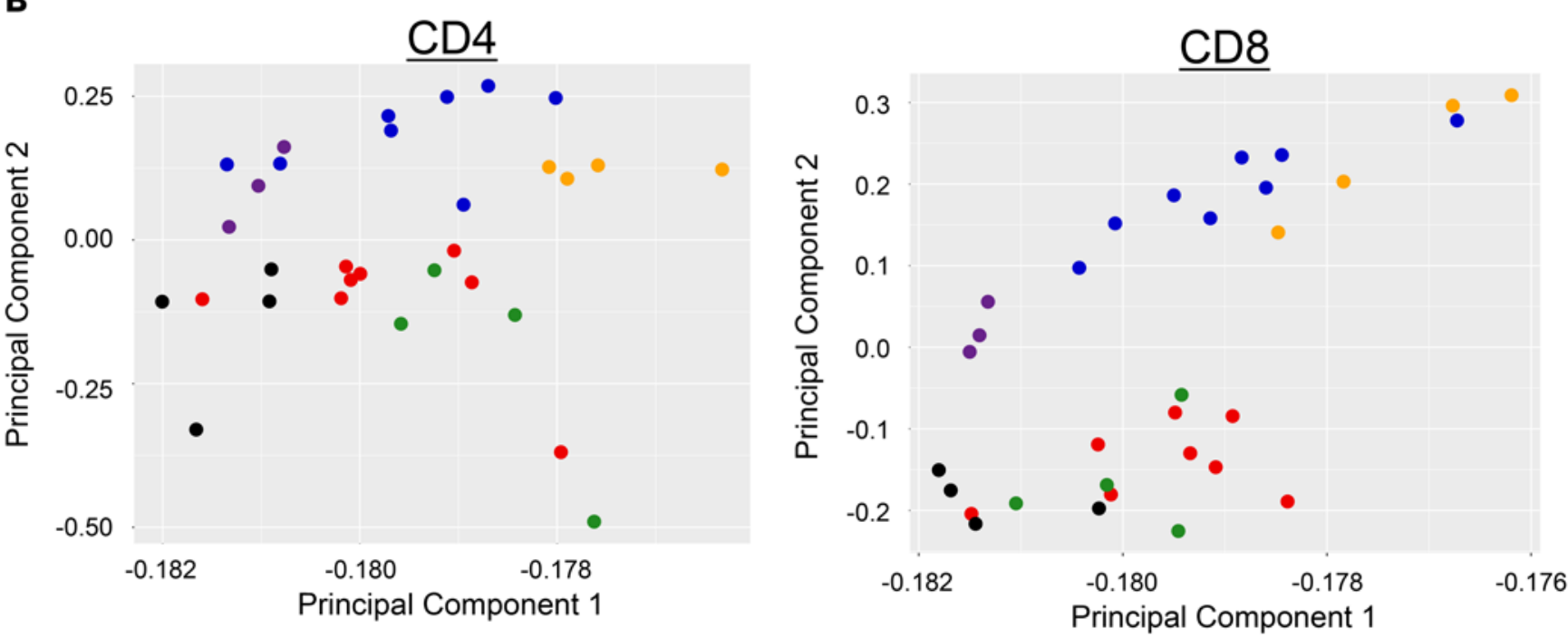

upregulation of transcription regulators, such as Txnip and Lef1, and cytokine and ligand receptors, such as Il6ra, Ifngr2, and Tnfrsf22, was observed in these groups (Figure 8). In addition to these factors, we found several hundred genes whose expression was significantly different at both time points (Figure 9A and Supplemental Tables 2 and 3). There was substantial conservation in gene expression in groups 2 and 4; however, there were also group-specific differences in gene expression, indicating a potential temporal aspect to the expression profiles related to the extent of time with decreased tumor burden (Figure 9A). In both $\mathrm{CD} 4^{+}$and $\mathrm{CD} 8^{+} \mathrm{T}$ cells, metabolic, biosynthetic, immune, proliferative, and cell death signatures were differentially expressed, although not to the same extent in the different subsets and changing slightly over time (Supplemental Table 4). These results describe a significant transcriptional reprogramming of tumor-infiltrating lymphocytes during a robust anti-PD-1-induced antitumor immune response.

We subsequently examined genes differentially regulated in samples in which tumor growth during anti-PD-1 therapy was observed, both early in treatment (group 3) and at later time points (group 6). We found a much more limited group of genes with expression that was significantly different at both time points when compared with their expression in mice with progressing tumors treated with IgG2a (Figure 9B and Supplemental Tables 2 and 3). In particular, gene expression of samples from group 3, harvested at the same time as those from the IgG2a-treated group 1, was remarkably similar to that observed in group 1 , while gene expression from samples harvested at later times (group 6) displayed higher deviation, yet 
CD4

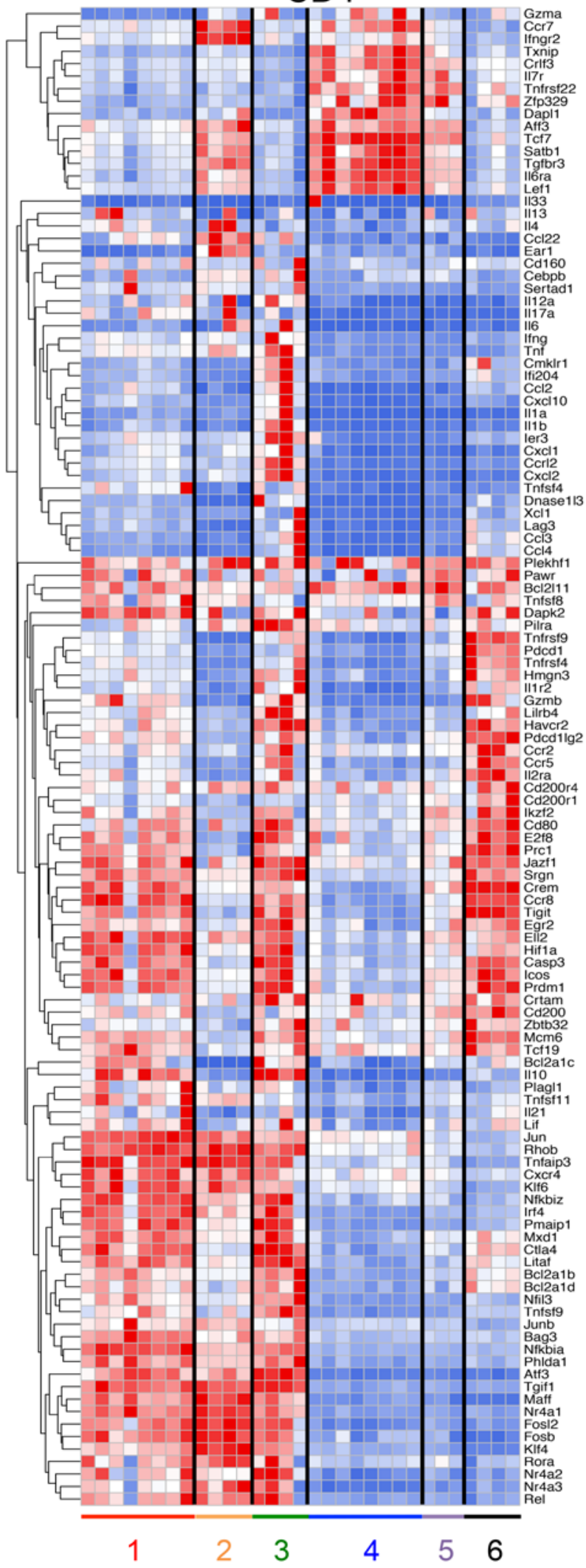

\section{CD8}

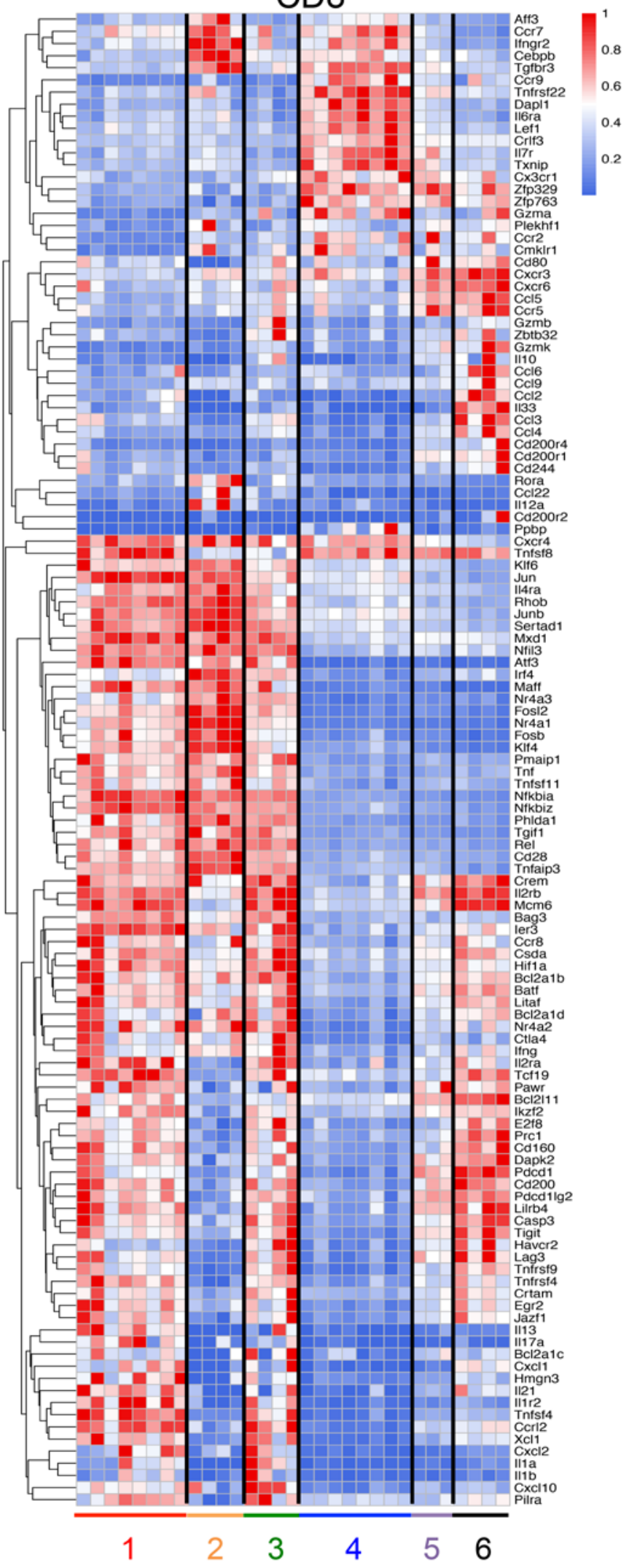


Figure 8. Heatmaps for differential gene expression. Heatmaps for differential gene expression in select transcriptional regulators, coinhibitory and costimulatory molecules, and cytokines and chemokines as well as their cognate receptors associated with exhaustion among the 6 groups in indicated cell types. Significance was set at $P<0.05$, adjusted $P<0.1$, and absolute $\log _{2}$ fold change $>1$. Moderated t-statistic from the limma package, with Benjamini and Hochberg's adjustment for multiple testings.

was still very similar (Figure 9B). In contrast to the transcriptional phenotype observed in groups 2 and 4 , coinhibitory molecules, such as Pdcd1, Havcr2, and Tigit; costimulatory molecules, such as Cd80, Tnfrsf4, and Tnfrsf9; and transcription regulators, such as $I k z f 2$, Prdm1, and Batf, displayed similar or progressively enhanced expression (Figure 8 and Supplemental Tables 2 and 3). While numbers of genes with significant differences in expression compared with that observed in IgG2a-treated samples were severely limited, those genes were highly enriched for upregulation of immune accumulation and response signatures in both $\mathrm{CD}^{+}$and $\mathrm{CD}^{+} \mathrm{T}$ cells (Supplemental Tables 3 and 4), involving such genes as $\mathrm{Ccl}$, Cxcl9, Ccl5, Ccr2, Ccr5, Gzma, Gzmb, and Gzmk, suggesting a transcriptional response to anti-PD-1 therapy promoting immune activity, albeit without therapeutic efficacy. Downregulated ontologies were primarily focused on immune differentiation in $\mathrm{CD} 4^{+}$and $\mathrm{CD} 8^{+} \mathrm{T}$ cells in group 3 and on metabolism and biosynthetic processes in $\mathrm{CD} 4^{+}$and $\mathrm{CD} 8^{+} \mathrm{T}$ cells in group 6 (Supplemental Table 4).

Groups 2 and 4 demonstrate a robust antitumor immune response, while groups 3 and 6 display a lack of therapeutic efficacy upon anti-PD-1 treatment. Group 5, however, demonstrated an intermediate tumor growth phenotype upon anti-PD-1 treatment, therefore we examined gene expression in those samples. In both $\mathrm{CD}^{+}$and $\mathrm{CD} 8^{+} \mathrm{T}$ cells, many key exhaustion-related genes, such as coinhibitory molecules, such as Pdcd1, Havcr2, and Tigit; costimulatory molecules, such as Cd80, Tnfrsf4, and Tnfrsf 9 ; and transcription regulators, such as $I k z f 2$, Prdm1, and Batf, displayed intermediate expression between that observed in regressing tumors (group 4) and that in outgrowing tumors (group 6) examined at the same time point (Figure 8). This observation was born out more globally: when compared with gene expression in IgG2a-treated controls, several hundred genes were differentially regulated in group 5 , with the majority of these also showing differential regulation in either regressing or outgrowing tumors (Figure 9C and Supplemental Figure 15). Gene overlap was slightly more skewed toward regressing than outgrowing tumors, although levels of overlap were similar and also more similar to those in samples harvested at the same time point, indicating a temporal aspect to the gene expression profile (Supplemental Figure 15). Prominent ontologies among differentially regulated genes between group 5 and IgG2a-treated mice included immune response, macromolecule synthesis, metabolism, proliferation, and regulation of cell death, with cell type-specific enrichment of different ontologies (Supplemental Table 4).

Anti-PD-1 therapy has been developed to restore functional capacities to exhausted $\mathrm{T}$ cells in the tumor microenvironment (4). Our data indicate that in early-stage NSCLC T lymphocytes exhibit cell type-specific functional cytokine production and differential gene expression upon therapy-induced tumor regression (Figures 5 and 8). We therefore performed gene set enrichment analyses to examine prevalence of exhaustion signatures in $\mathrm{CD}^{+}$and $\mathrm{CD} 8^{+} \mathrm{T}$ cells isolated from tumors with different degrees of response to anti-PD-1. As expected, $\mathrm{CD} 4^{+} \mathrm{T}$ cells from samples with robust tumor regression (groups 2 and 4 ) showed a significantly depleted exhaustion signature when compared with their counterparts from IgG2a-treated mice (Figure 10). This depleted exhaustion signature in $\mathrm{CD} 4^{+} \mathrm{T}$ cells was also observed in samples from intermediate tumor growth phenotypes (group 5), while samples from outgrowing tumors (groups 3 and 6) had either trends for enhancement of the exhaustion signature or no significant modulation of the signature (Figure 10). $\mathrm{CD} 8^{+} \mathrm{T}$ cells followed a similar pattern in gene set enrichment but at less significant levels than their $\mathrm{CD} 4^{+}$counterparts (Figure 10). We further examined differential gene expression observed between these groups and IgG2a-treated mice by comparing these signatures to those of naive, effector, memory, and exhausted $\mathrm{CD} 4^{+}$and $\mathrm{CD} 8^{+} \mathrm{T}$ cells $(28) . \mathrm{CD} 4^{+} \mathrm{T}$ cells from regressing and intermediate tumor growth phenotypes showed a skewing away from exhausted- and effector-like signatures toward more naive- or memory-like signatures when compared with IgG2a-treated mice, while those from outgrowing tumors skewed more toward an effector-like phenotype than naive- or memory-like signatures when compared with IgG2a-treated mice (Supplemental Table 5). This skewing was not replicated in the $\mathrm{CD} 8^{+} \mathrm{T}$ cells: the primary observed gene set enrichment showed a more naive phenotype than an exhausted, effector, or memory phenotype in group 4 when compared with IgG2a (Supplemental Table 5).

In aggregate, these data suggest that anti-PD-1 therapy induces robust and cell type-specific changes to the transcriptome of $\mathrm{CD}^{+}$and $\mathrm{CD} 8^{+} \mathrm{T}$ cells in the tumor microenvironment corresponding to the degree of antitumor response. 
A

\section{CD4}

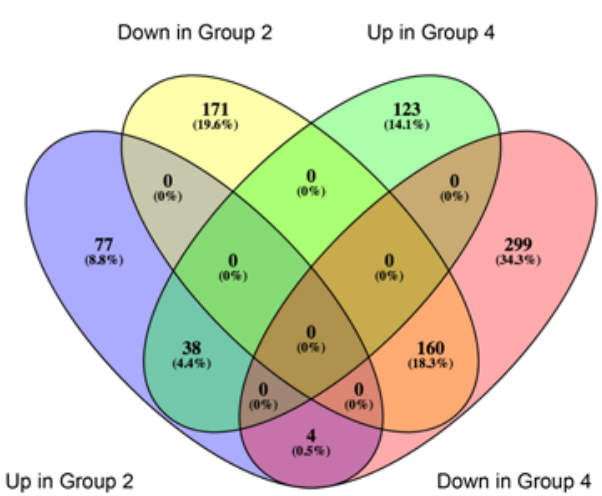

B

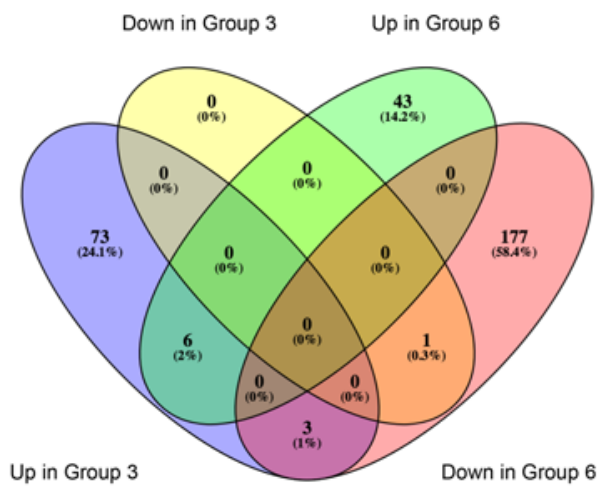

C

Down in Group 2 or $4 \quad$ Up in Group 5

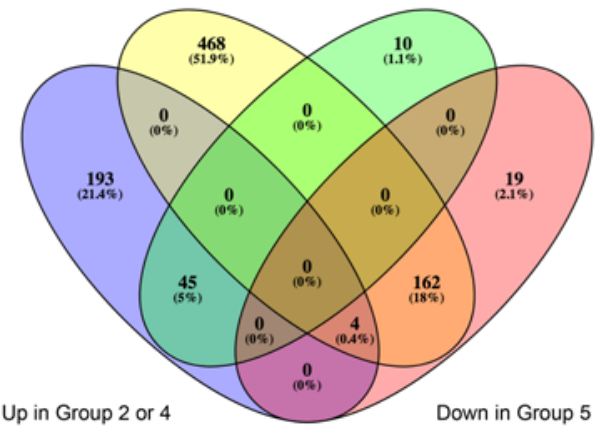

Down in Group 3 or 6

Up in Group 5

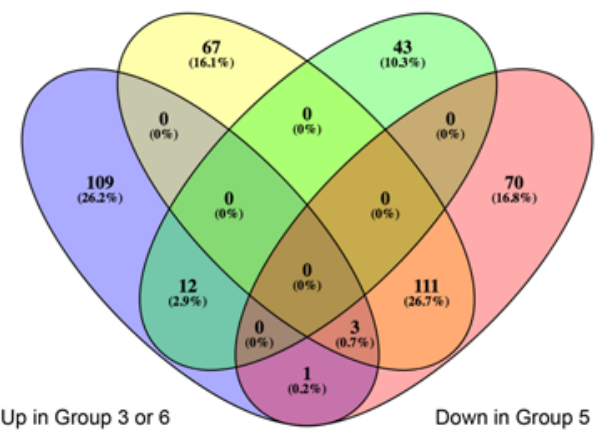

CD8
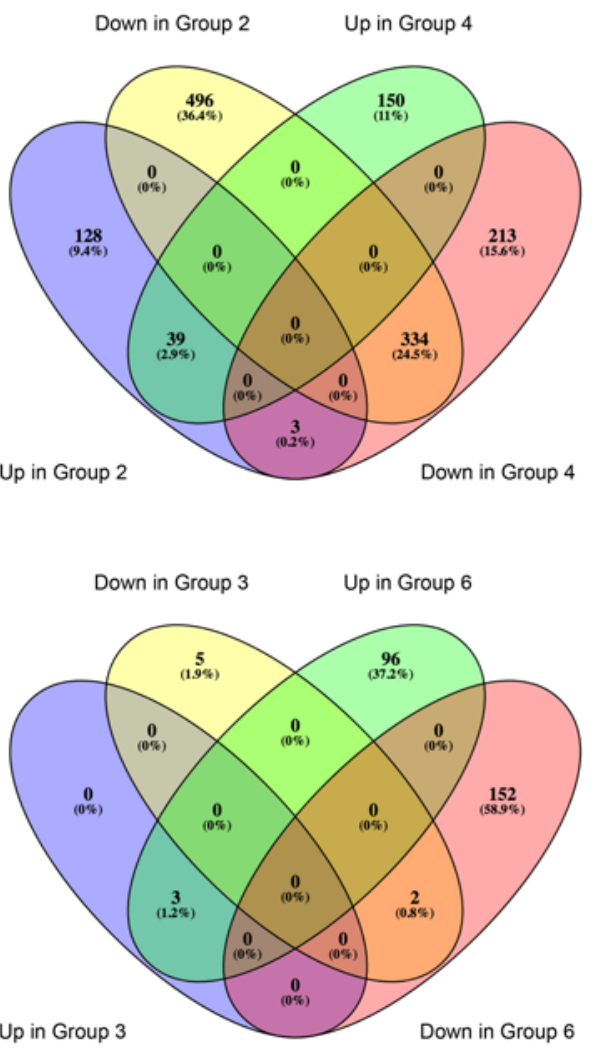

Down in Group 2 or $4 \quad$ Up in Group 5

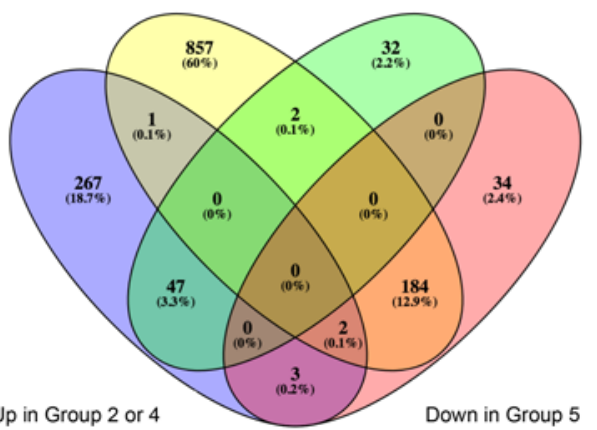

Down in Group 3 or $6 \quad$ Up in Group 5

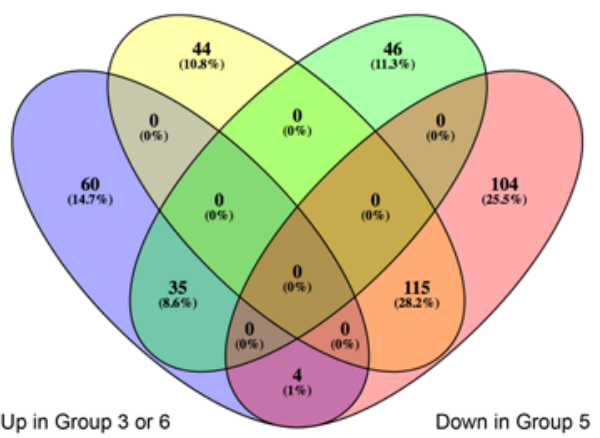

Figure 9. Differential gene expression of tumor-infiltrating $T$ cell subsets upon antiPD-1 treatment in HKP1 lung cancer model. Venn diagrams of overlap in differentially regulated genes compared with their expression in IgG2a between those upregulated and downregulated in $\mathrm{CD}^{+}$ and $\mathrm{CD} 8^{+} \mathrm{T}$ cells in groups 2 and 4 (A), groups 3 and 6 (B), and group 5 compared with groups 2 and 4 or groups 3 and 6 (C). 


\section{Discussion}

Given the unexplored potential of leveraging immunotherapeutics at early stages in NSCLC progression, in this study we sought to evaluate the therapeutic effect and underlying cellular and molecular mechanisms of the early immune response to targeted inhibition of the PD-1/PD-L1 axis. Analysis of early-stage NSCLC patients (stages I-IIIA) showed significant immune activity, as characterized by robust $\mathrm{PD}-\mathrm{L} 1$ expression associated with marked infiltration of $\mathrm{PD}-1^{+} \mathrm{T}$ cells in the tumor microenvironment. Indeed, this accumulation of $\mathrm{PD}-1^{+} \mathrm{T}$ cells in the tumor was observed in both $\mathrm{CD} 4^{+}$and $\mathrm{CD} 8^{+}$ $\mathrm{T}$ cells over the spectrum of stages analyzed in patient tissues (Supplemental Figure 16). These clinical features were faithfully recapitulated in early tumors in the orthotopic HKP1 model, leading us to utilize this model to target the PD-1/PD-L1 axis in early-stage lung cancer. Indeed, expression of PD-L1 in tumor cells and infiltrating immune cells and PD-1 in tumor-infiltrating T cells has been associated with responsiveness to blockade of the PD-1/PD-L1 immune checkpoint in NSCLC across the spectrum of the disease $(8,9,29-32)$. Anti-PD-1 monotherapy yielded significant tumor control and improved survival in mice, which was associated with a robust antitumor immune response, as revealed by enhanced proliferation of $\mathrm{T}$ cell subsets and increased expression of effector cytokines by infiltrated $\mathrm{CD}^{+} \mathrm{T}$ cells. Depletion of $\mathrm{CD}^{+}$and $\mathrm{CD} 8^{+} \mathrm{T}$ cells resulted in diminished efficacy of anti-PD-1 therapy, with strong synergistic effects between the two populations modulating tumor growth. Analysis of the transcriptome of lung-infiltrating $\mathrm{CD}^{+}$and $\mathrm{CD}^{+} \mathrm{T}$ cells from tumor-bearing mice revealed differential gene expression corresponding to the degree of antitumor response.

Our findings demonstrating the efficacy of single-agent anti-PD-1 therapy in orthotopic HKP1 tumors differ from those of previous reports that show marked unresponsiveness in the KP genetically engineered mouse model (GEMM) of spontaneous lung adenocarcinoma $(33,34)$. Indeed, whole exome sequencing uncovered a substantially higher mutational burden in HKP1 orthotopic tumors compared with the autochthonous KP tumors (Supplemental Figure 17, A and B). Furthermore, analysis of a data set from the The Cancer Genome Atlas generated from comprehensive molecular profiling of 585 resected lung adenocarcinomas (originally described in ref. 35) showed that patients with early-stage (I/II) Kras mutant NSCLC show somatic mutation rates much higher than those seen in autochthonous KP tumors; in particular, early-stage nonsmokers from this data set had mutation rates comparable to those observed in HKP1 orthotopic tumors (Supplemental Figure 17, A and B). It is possible that responsiveness of HKP1 tumors to anti-PD-1 treatment may be attributed in part to the higher mutational load, which has been recognized as an important determinant of the response to immune checkpoint inhibitors, including PD-1/PD-L1 inhibition in NSCLC patients (36-38). Consistent with our findings, low mutation burden in autochthonous $\mathrm{KP}$ tumors has been recently reported $(39,40)$ and is also associated with low to nonexistent lymphocytic infiltration $(34,41)$. Indeed, lack of infiltrating $\mathrm{T}$ cells in the tumor beds is considered a major barrier to responsiveness to PD-1 blockade $(12,14)$. As expected, the higher mutational burden in HKP1 tumors was associated with tumor infiltration by $\mathrm{CD}^{+} \mathrm{T}$ lymphocytes.

Anti-PD-1 treatment initiated during this early phase of tumor growth yielded markedly increased proliferation of both $\mathrm{CD}^{+}$and $\mathrm{CD}^{+} \mathrm{T}$ cells; however, elevated expression of effector cytokines IFN- $\gamma$ and TNF- $\alpha$ was unexpectedly confined to $\mathrm{CD}^{+} \mathrm{T}$ cells and not observed in $\mathrm{CD} 8^{+} \mathrm{T}$ cells. Similar to our results, proliferation of $\mathrm{T}$ cells has been observed in the peripheral blood of patients after PD-1-targeted therapy in lung cancer patients, with the most robust proliferative increase being observed in $\mathrm{CD}^{+} \mathrm{T}$ cells; this proliferative effect strongly correlates with clinical response, emphasizing the well-established potent antitumor efficacy of $\mathrm{CD}^{+} \mathrm{T}$ cells (42). The effect of anti-PD-1 therapy on CD4 ${ }^{+} \mathrm{T}$ cells, however, seems to be more disease specific $(11,13,23,28,43-45)$. Recent work has shown reduced Treg frequencies upon anti-PD-1 therapy in colorectal and melanoma models (46), while our data indicate increased proliferation in both Tregs and Th cells and increased cytokine production in $\mathrm{CD}^{+} \mathrm{T}$ cells after anti-PD-1 blockade in early-stage NSCLC. Also of note was the marked enhancement in PD-1 expression in Tregs. This corresponded with a greater pool of PD- $1^{+}$Tregs, which produced increased IFN- $\gamma$ compared with PD- $1^{-}$Tregs. These data suggest a dysfunctional aspect to these cells, similar to previous reports, which could result in antitumor activity (47). These cytokine effects in $\mathrm{CD} 4^{+} \mathrm{T}$ cells may mediate the therapeutic efficacy of the treatment, mirroring effects in breast cancer, in which effective immunotherapy has been shown to require robust $\mathrm{CD}^{+} \mathrm{T}$ cell responses (48) and to possibly potentiate antitumor $\mathrm{CD}^{+} \mathrm{T}$ cell activity. Indeed, cumulative IFN- $\gamma$ and TNF- $\alpha$ production from CD4 ${ }^{+} \mathrm{T}$ cells inversely correlated with tumor burden (Supplemental Figure 18) $(49,50)$. Most importantly, depletion of either $\mathrm{CD}^{+}$or $\mathrm{CD}^{+} \mathrm{T}$ cells alone reduced anti-PD-1 efficacy, demonstrating their individual roles in the antitumor response. Importantly, a marked 


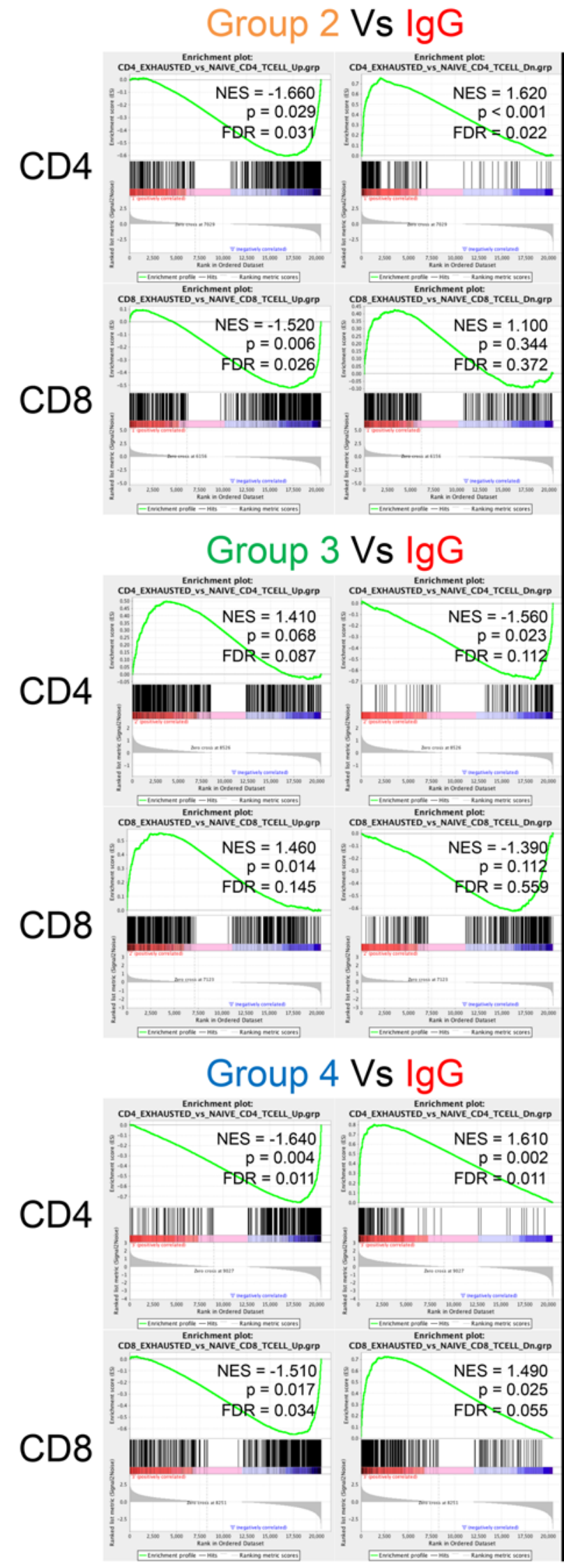

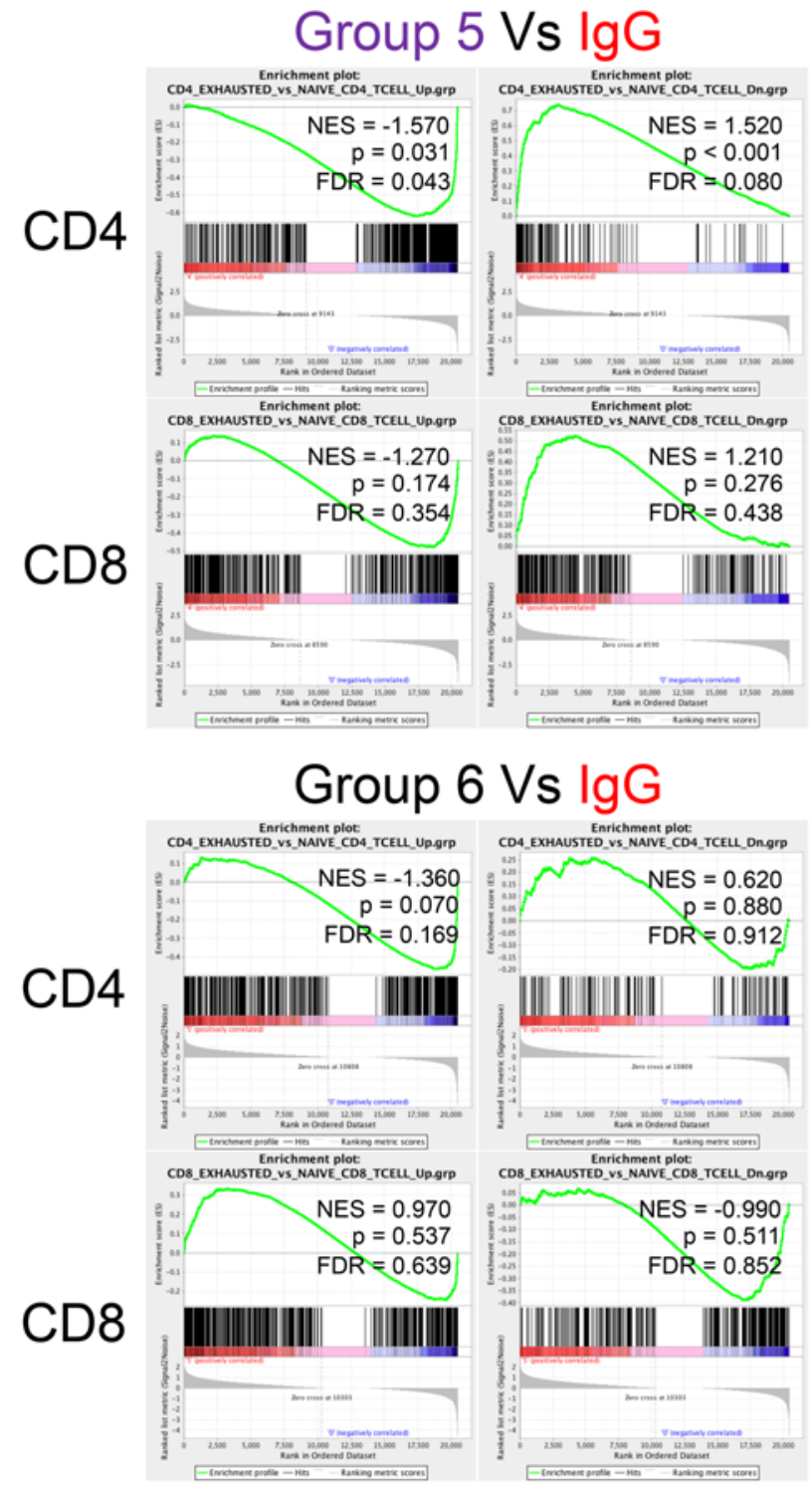

Figure 10. Hyperparametric plots examining enrichment of exhaustion-associated gene sets. Hyperparametric plots examining enrichment of gene sets in groups $2-6$ compared with IgC2a (group 1) in $\mathrm{CD}^{+}$ $T$ cells or $\mathrm{CD}^{+} \mathrm{T}$ cells for genes enriched in exhausted T cells compared with naive T cells (GEO accession GSE30431). Normalized enrichment scores (NES), $P$ values, and false discovery rates (FDR) are shown. Hypergeometric $P$ values from the GOstats package. 
synergistic effect following simultaneous depletion of $\mathrm{CD}^{+}$and $\mathrm{CD} 8^{+} \mathrm{T}$ cells emphasizes the potent interplay between these populations in modulating lung cancer progression and affecting anti-PD-1 response.

Our transcriptome analysis of T cells isolated from lungs during and after anti-PD-1 therapy revealed several interesting phenomena. First, gene expression correlated more strongly with tumor burden than treatment status, as indicated by principal component distributions and gene expression profiles, similar to that observed in a breast cancer model (48). Comparisons between IgG2a- and anti-PD-1-treated samples harvested at day 14 display this to great effect: anti-PD-1-treated samples with substantial regression (group 2) have markedly distinct gene expression profiles, while anti-PD-1-treated samples with a less robust effect on tumor burden (group 3) have significantly less variation in gene expression from the IgG2a-treated samples. However, it should be noted that the cohort of genes differentially expressed in these group 3 samples in both the $\mathrm{CD}^{+}$and $\mathrm{CD}^{+} \mathrm{T}$ cell subsets are enriched for immune function-related ontologies, indicating anti-PD-1 activity, even without clear antitumor efficacy. Second, the exhaustion signature in $\mathrm{CD}^{+} \mathrm{T}$ cells is rapidly lost in samples in which there is tumor regression, while in tumors with progression under anti-PD-1 therapy, there is no effect or even a slight enhancement of the signature by gene set enrichment analysis (Figure 10). The same pattern is followed in $\mathrm{CD} 8^{+} \mathrm{T}$ cells but much less significantly. Third, the gene expression profiles in lung-infiltrating lymphocytes are time dependent in addition to treatment and tumor burden dependent. Our experimental design sampled T cells at multiple stages in the antitumor response, and while there was significant overlap in gene expression between samples, with similar tumor growth phenotypes, there were substantial discrepancies as well. While this could potentially be attributed in part to cessation of anti-PD-1 treatment on day 16, samples harvested at days 17 and 24 were practically indistinguishable. Finally, samples from mice with an intermediate tumor growth phenotype displayed an intermediate gene expression profile, potentially indicating a continuum of response to anti-PD-1 therapy.

Importantly, anti-PD-1 therapy resulted in a reprogramming of multiple immune subsets in the tumor microenvironment, including a significant decrease in PMN-MDSCs (51). Furthermore, the myeloid compartment displayed an accumulation of mature macrophages with reduced IDO and increased NOS2 production, potentially favoring a more M1-like tumoricidal phenotype and mirroring recent data in colorectal cancer models showing an antitumor effect mediated by tumor-associated macrophages following anti-PD-1 treatment $(24,52)$. However, we also observed a global increase in NOS2 production in all myeloid subsets and increased production of IDO in Mo-MDSCs. Both NOS2 and IDO have been shown to limit T cell growth and promote $\mathrm{T}$ cell apoptosis. NOS2, while being a marker for M1-like macrophages, has immunosuppressive functionalities in the context of other enzymes, such as arginase $1(52,53)$. NOS2 induces nitric oxide production, which blocks IL-2 receptor signaling $(52,53)$. IDO oxidatively catabolizes tryptophan, depleting L-tryptophan and producing its metabolites, while also altering redox potentials through superoxide radical consumption $(52,53)$. This production of IDO in Mo-MDSCs upon treatment was of particular interest, as this mirrored the increased IDO production at 2 weeks in treatment-naive mice; in both contexts, $\mathrm{T}$ cell proliferation and cytokine production were comparatively elevated, suggesting that IDO production may be an integral negative regulator of adaptive immunity in this system and a potential target for therapeutic intervention. The enrichment of these negative feedback mechanisms upon PD-1 blockade could be a consequence of the enhanced inflammatory microenvironment induced by the blockade and could potentially limit the efficacy of the therapy. The functional implications of these observations necessitate further study. PD-1 blockade increased proliferation of Tregs in addition to Th and $\mathrm{CD}^{+}$cells; while this increased presence of immunosuppressive Tregs could be involved in the eventual failure of anti-PD-1 monotherapy, there was also an accumulation of IFN- $\gamma$-producing Tregs, a population that may enhance anti-PD-1's antitumor efficacy. The potential therapeutic implications of anti-PD-1-mediated reprogramming of the immune microenvironment in early-stage NSCLC warrant further exploration.

The potential efficacy of treating early-stage lung cancer was recently demonstrated in a pilot study examining neoadjuvant nivolumab administration prior to surgical resection in patients with untreated stage I, II, or IIIA NSCLC (38). The authors found induction of a major pathological response in the resected tissue in $45 \%$ of patients that was associated with increased mutation burden. Importantly, treatment with nivolumab resulted in expansion of neoantigen-specific $\mathrm{T}$ cells in peripheral blood; these clones could also be found in resected tumor tissue and involved lymph nodes. These results clearly demonstrate the potential of targeting these patients, who still have a relatively poor prognosis (5-year survival ranging from $50 \%$ at stage IA to $20 \%$ at stage IIIA and frequent relapse after resection) but have a more intact immune system than advanced-disease patients (38). Our studies provide further mechanistic insights into 
responses of these early-stage tumors to treatment, indicating both functional characteristics and potential avenues for synergistic approaches; they may aid in the design and implementation of future neoadjuvant immunotherapy trials in NSCLC, such as a recently initiated open-label, single-arm prospective phase II neoadjuvant anti PD-1 immunotherapy trial in resectable NSCLC (ClinicalTrials.gov NCT03197467; https://clinicaltrials.gov/ct2/show/NCT03197467).

While we observed that pharmacological inhibition with a single-agent anti-PD-1 antibody resulted in significant tumor control and improved survival in mice, associated with a robust antitumor immune response, tumor outgrowth was observed upon cessation of treatment (Figure 4, A and B). In this context, adaptive resistance to therapeutic PD-1/PD-L1 blockade has been recently encountered (54, 55); however, very little is known regarding the underlying mechanisms that govern resistance (56). Recently, upregulation of another inhibitory checkpoint receptor, TIM-3, was associated with resistance to anti-PD-1 therapy in the Kras GEMM, and TIM-3 targeting extended the benefit of PD-1 blockade (33). We have observed increased expression of both $2 \mathrm{~B} 4$ and LAG-3 on $\mathrm{CD}^{+} \mathrm{T}$ cells at later stages in the HKP1 model and increased mRNA expression of several other checkpoint proteins, such as Havcr2, Cd200r1, and Cd200r4, on CD4 $4^{+}$and CD8 ${ }^{+} \mathrm{T}$ cells in samples with a less effective response to anti-PD-1 therapy, suggesting the potential of combinatorial therapeutics to enhance anti-PD-1 efficacy and elicit more durable responses. In summary, our preclinical study mechanistically provides evidence for targeting the PD-1/PD-L1 axis in early-stage NSCLC, including targeting the mutant Kras cohort that is impervious to available targeted therapies.

\section{Methods}

A detailed description of materials and methods, including a list of antibodies and recombinant proteins used, can be found in the Supplemental Methods.

Patient sample collection and use. Tissue for immunofluorescence, IHC, and histological analyses was washed, fixed, and embedded in either OCT for immunofluorescence or paraffin for IHC and histological analyses. Four- to ten- $\mu \mathrm{m}$-thick sections were cut and stained using standard protocols. Tissue for flow cytometry analyses was minced, digested with collagenase and DNAse, strained through $40-\mu \mathrm{m}$ filters, and red blood cells lysed. Single-cell suspensions were stained and analyzed using standard methodologies. Please see the Supplemental Methods for detailed methodologies, including antibody details.

Animal work. Spontaneous $K R A S^{\mathrm{LSL}-\mathrm{G} 12 \mathrm{D} /+} ; T P 53^{\text {flox/flox }}(\mathrm{KP})$ mice were generated and tumors were induced using intratracheal adenoviral administration as described previously (25). HKP1 lung cancer cells derived from KP tumor lungs and expressing mCherry-luciferase were generated as described previously (25), and orthotopic tumors in the lungs were generated via tail vein administration of 150,000 cells in $100 \mu \mathrm{l}$ sterile PBS into 8-week-old female C57BL6/J mice. Bioluminescence imaging was conducted twice weekly. For treatment studies, $250 \mu \mathrm{g}$ of either anti-PD-1 (clone RMP1-14, BioXCell) or IgG2a control (clone 2A3, BioXCell) was diluted in sterile PBS and administered intraperitoneally in a volume of $100 \mu \mathrm{l}$ per dose on either days $6,10,13$, and 17 or days 7, 10, 13, and 16 after implantation. For depletion studies, matched cohorts of mice were treated on day 6 with $400 \mu \mathrm{g}$ anti-CD4 (clone GK1.5, BioXCell) + $400 \mu \mathrm{g}$ IgG2b (clone LTF-2, BioXCell), $400 \mu \mathrm{g}$ anti-CD8a (clone 2.43, BioXCell) + $400 \mu \mathrm{g}$ IgG2b, $400 \mu \mathrm{g}$ anti-CD4 + $400 \mu \mathrm{g}$ anti-CD8a, or $800 \mu \mathrm{g}$ IgG2b; mice were subsequently treated with half doses of antibody or IgG2b control on days 9, 12, and 15 and with $250 \mu \mathrm{g} /$ dose of anti-PD-1 on days 7, 10, 13, and 16. At sacrifice, mice were euthanized and perfused with PBS and lungs were extracted. Tissue for immunofluorescence and histological analyses was washed, fixed, and embedded in OCT. Eight- to ten- $\mu$ m-thick sections were cut and stained using standard protocols. Tissue for flow cytometry analyses was diced, ground through a $140-\mu \mathrm{m}$ mesh, and filtered through a $70-\mu \mathrm{m}$ filter, and red blood cells were lysed. Single-cell suspensions were stained using standard protocols. Stains requiring stimulation were treated with PMA, ionomycin, and Golgi blockers for 4 hours and subsequently stained and analyzed using standard methodologies. Please see the Supplemental Methods for detailed methodologies, including antibody details. Tissue for RNA-sequencing analyses was diced, ground through a $140-\mu \mathrm{m}$ mesh, and filtered through a $70-\mu \mathrm{m}$ filter, and red blood cells were lysed. Single-cell suspensions were stained using standard protocols. Cells were sorted via flow cytometry into lysis buffer. RNA was extracted using the RNeasy kit with on-column DNA digestion and submitted for sequencing. Library preparation was performed using the Illumina TruSeq RNA Sample Preparation kit, and 8 samples per lane were sequenced with single-end 50 bps on a HiSeq4000 (Illumina). Expression analysis and group comparisons were performed using mouse genome build $\mathrm{mm} 9$ and standard data analysis tools; please 
see the Supplemental Methods for detailed methodologies. Tissue for whole exome sequencing analyses was obtained from grossly visible nodules or regions known to contain tumor by bioluminescence imaging. Germline DNA was extracted from blood in the inferior vena cava. DNA was extracted using the DNeasy kit and submitted for sequencing. Library preparation was performed using the SureSelect kit (Agilent). Eight samples were run on the HiSeq4000 with paired-end clustering and 2 rounds of 100 cycles. Mutation analysis was performed using mouse genome build mm10 and standard data analysis tools; please see the Supplemental Methods for detailed methodologies.

Accession numbers. The RNA sequencing data are available in the Gene Expression Omnibus database (http://www.ncbi.nlm.nih.gov/gds) under the accession number GSE114300. The previously published data set from Crawford et al. (28), which contains transcriptome data from naive, memory, effector, and exhausted $\mathrm{CD}^{+}$and $\mathrm{CD} 8^{+} \mathrm{T}$ cells that we compared with our RNA sequencing data, is available under the accession number GSE30431. Whole exome sequencing data are available in the BioProject database (http://www.ncbi.nlm.nih.gov/bioproject) under the project number PRJNA470948.

Statistics. Results are expressed as mean \pm SEM. Analyses of different time points in untreated tumor progression were performed using 1- or 2-way ANOVA with Tukey's multiple comparisons test. Analyses of different treatment groups were performed using the log-rank (Mantel-Cox) test for survival and 2-tailed $t$ tests corrected for multiple comparisons when appropriate using the Holm-Sidak method, using the GraphPad Prism statistical program. $P$ values of less than 0.05 were considered significant.

Study approval. All patient samples were obtained following informed consent at the Cardiothoracic Surgery Department of Weill Cornell Medical College under the Thoracic Surgery Biobank protocol 1008011221, which was approved by the Institutional Review Board at Weill Cornell Medical College. All animal work was performed in accordance with an animal protocol approved by the Institutional Animal Care and Use Committee at Weill Cornell Medical College (protocol 0806-762A).

\section{Author contributions}

GJM, LSH, STCW, DG, NKA, and VM conceived the experiments. GJM and VM designed all the experiments with input from other authors. YB, SBL, and JST performed human tissue immunostaining. NN performed all pathological analyses. MJPC performed mouse tissue immunostaining. GJM and LSH performed baseline mouse tumor immune analysis. GJM, LSH, and MJPC performed in vivo mouse treatment studies. GJM, MJPC, and STCW generated and analyzed lung-infiltrating T cell RNA sequencing analyses. BB and OE performed mutational analyses on mouse tumor samples and The Cancer Genome Atlas data sets. GJM and VM wrote this paper with feedback from all authors.

\section{Acknowledgments}

We thank Jenny Xiang of the Weill Cornell Medicine Genomics Resources Core Facility and Jason McCormick of the Weill Cornell Medicine Flow Cytometry Core Facility for expert advice on experimental design and analysis as well as aid in experimental execution. We are also thankful for generous funds donated by patients in the Division of Thoracic Surgery to NKA. This work was supported by National Cancer Institute (NCI) grant NIH U01 CA188388 (to VM and STCW) and NCI grant NIH T32 CA203702 (to GJM).

Address correspondence to: Vivek Mittal, 1300 York Avenue, A603, New York, New York 10065, USA. Phone: 212.746.9401; Email: vim2010@med.cornell.edu. Or to: Nasser Altorki, 525 E. 68th Street, M-404, New York, New York 10065, USA. Phone: 212.746.5156; Email: nkaltork@med.cornell.edu.

LSH's present address is: Regeneron, Tarrytown, New York, USA.

JST's present address is: Stony Brook University, New York, New York, USA.

NN's present address is: NYU Langone Health, New York, New York, USA.

1. Siegel RL, Miller KD, Jemal A. Cancer statistics, 2017. CA Cancer J Clin. 2017;67(1):7-30.

2. Torre LA, Siegel RL, Jemal A. Lung cancer statistics. Adv Exp Med Biol. 2016;893:1-19.

3. Pao W, Hutchinson KE. Chipping away at the lung cancer genome. Nat Med. 2012;18(3):349-351.

4. Pardoll DM. The blockade of immune checkpoints in cancer immunotherapy. Nat Rev Cancer. 2012;12(4):252-264. 
5. Couzin-Frankel J. Breakthrough of the year 2013. Cancer immunotherapy. Science. 2013;342(6165):1432-1433.

6. Zavala VA, Kalergis AM. New clinical advances in immunotherapy for the treatment of solid tumours. Immunology. 2015;145(2):182-201.

7. Brahmer JR, et al. Safety and activity of anti-PD-L1 antibody in patients with advanced cancer. $N$ Engl JMed. 2012;366(26):2455-2465.

8. Topalian SL, et al. Safety, activity, and immune correlates of anti-PD-1 antibody in cancer. N Engl J Med. 2012;366(26):2443-2454.

9. Gettinger SN, et al. Overall survival and long-term safety of nivolumab (anti-programmed death 1 antibody, BMS-936558, ONO-4538) in patients with previously treated advanced non-small-cell lung cancer. J Clin Oncol. 2015;33(18):2004-2012.

10. Reck M, et al. Pembrolizumab versus chemotherapy for PD-L1-positive non-small-cell lung cancer. $N$ Engl J Med. 2016;375(19):1823-1833.

11. Schietinger A, Greenberg PD. Tolerance and exhaustion: defining mechanisms of T cell dysfunction. Trends Immunol. 2014;35(2):51-60

12. Joyce JA, Fearon DT. T cell exclusion, immune privilege, and the tumor microenvironment. Science. 2015;348(6230):74-80.

13. Wherry EJ, Kurachi M. Molecular and cellular insights into T cell exhaustion. Nat Rev Immunol. 2015;15(8):486-499.

14. Spranger S. Mechanisms of tumor escape in the context of the T-cell-inflamed and the non-T-cell-inflamed tumor microenvironment. Int Immunol. 2016;28(8):383-391.

15. Sharma P, Hu-Lieskovan S, Wargo JA, Ribas A. Primary, adaptive, and acquired resistance to cancer immunotherapy. Cell. 2017;168(4):707-723

16. National Lung Screening Trial Research Team, et al. Reduced lung-cancer mortality with low-dose computed tomographic screening. N Engl J Med. 2011;365(5):395-409.

17. Black WC, et al. Cost-effectiveness of CT screening in the National Lung Screening Trial. N Engl J Med. 2014;371(19):1793-1802.

18. Siegel R, Ma J, Zou Z, Jemal A. Cancer statistics, 2014. CA Cancer J Clin. 2014;64(1):9-29.

19. Agata $\mathrm{Y}$, et al. Expression of the PD-1 antigen on the surface of stimulated mouse $\mathrm{T}$ and B lymphocytes. Int Immunol. 1996;8(5):765-772.

20. Keir ME, Butte MJ, Freeman GJ, Sharpe AH. PD-1 and its ligands in tolerance and immunity. Annu Rev Immunol. 2008;26:677-704.

21. Carter L, et al. PD-1:PD-L inhibitory pathway affects both CD4(+) and CD8(+) T cells and is overcome by IL-2. Eur J Immunol. 2002;32(3):634-643.

22. Nurieva R, et al. T-cell tolerance or function is determined by combinatorial costimulatory signals. EMBO J. 2006;25(11):2623-2633

23. Pauken KE, Wherry EJ. Overcoming T cell exhaustion in infection and cancer. Trends Immunol. 2015;36(4):265-276.

24. Gordon SR, et al. PD-1 expression by tumour-associated macrophages inhibits phagocytosis and tumour immunity. Nature. 2017;545(7655):495-499.

25. Choi $\mathrm{H}$, et al. Transcriptome analysis of individual stromal cell populations identifies stroma-tumor crosstalk in mouse lung cancer model. Cell Rep. 2015;10(7):1187-1201.

26. De Henau O, et al. Overcoming resistance to checkpoint blockade therapy by targeting PI3K $\gamma$ in myeloid cells. Nature. 2016;539(7629):443-447.

27. Engblom C, Pfirschke C, Pittet MJ. The role of myeloid cells in cancer therapies. Nat Rev Cancer. 2016;16(7):447-462.

28. Crawford A, et al. Molecular and transcriptional basis of $\mathrm{CD}^{+} \mathrm{T}$ cell dysfunction during chronic infection. Immunity. 2014;40(2):289-302.

29. Herbst RS, et al. Predictive correlates of response to the anti-PD-L1 antibody MPDL3280A in cancer patients. Nature. 2014;515(7528):563-567.

30. Taube JM, et al. Association of PD-1, PD-1 ligands, and other features of the tumor immune microenvironment with response to anti-PD-1 therapy. Clin Cancer Res. 2014;20(19):5064-5074.

31. Mazzaschi G, et al. Low PD-1 expression in cytotoxic CD8+ tumor-infiltrating lymphocytes confers an immune-privileged tissue microenvironment in NSCLC with a prognostic and predictive value. Clin Cancer Res. 2018;24(2):407-419.

32. Wu SP, et al. Stromal PD-L1-positive regulatory T cells and PD-1-positive CD8-positive T cells define the response of different subsets of non-small cell lung cancer to PD-1/PD-L1 blockade immunotherapy. J Thorac Oncol. 2018;13(4):521-532.

33. Koyama S, et al. Adaptive resistance to therapeutic PD-1 blockade is associated with upregulation of alternative immune checkpoints. Nat Commun. 2016;7:10501.

34. Pfirschke C, et al. Immunogenic chemotherapy sensitizes tumors to checkpoint blockade therapy. Immunity. 2016;44(2):343-354.

35. Cancer Genome Atlas Research Network. Comprehensive molecular profiling of lung adenocarcinoma. Nature. 2014;511(7511):543-550.

36. Rizvi NA, et al. Cancer immunology. Mutational landscape determines sensitivity to PD-1 blockade in non-small cell lung cancer. Science. 2015;348(6230):124-128.

37. Schumacher TN, Schreiber RD. Neoantigens in cancer immunotherapy. Science. 2015;348(6230):69-74.

38. Forde PM, et al. Neoadjuvant PD-1 blockade in resectable lung cancer. N Engl J Med. 2018;378(21):1976-1986.

39. Westcott PM, et al. The mutational landscapes of genetic and chemical models of Kras-driven lung cancer. Nature. 2015;517(7535):489-492.

40. McFadden DG, et al. Mutational landscape of EGFR-, MYC-, and Kras-driven genetically engineered mouse models of lung adenocarcinoma. Proc Natl Acad Sci USA. 2016;113(42):E6409-E6417.

41. DuPage M, et al. Endogenous $\mathrm{T}$ cell responses to antigens expressed in lung adenocarcinomas delay malignant tumor progression. Cancer Cell. 2011;19(1):72-85.

42. Kamphorst AO, et al. Proliferation of PD-1+ CD8 T cells in peripheral blood after PD-1-targeted therapy in lung cancer patients. Proc Natl Acad Sci USA. 2017;114(19):4993-4998

43. Pauken KE, et al. Epigenetic stability of exhausted T cells limits durability of reinvigoration by PD-1 blockade. Science. 2016;354(6316):1160-1165.

44. Schietinger A, et al. Tumor-specific T cell dysfunction is a dynamic antigen-driven differentiation program initiated early during tumorigenesis. Immunity. 2016;45(2):389-401.

45. Sen DR, et al. The epigenetic landscape of T cell exhaustion. Science. 2016;354(6316):1165-1169. 
46. Wei SC, et al. Distinct cellular mechanisms underlie anti-CTLA-4 and anti-PD-1 checkpoint blockade. Cell. 2017;170(6):1120-1133.e17.

47. Lowther DE, et al. PD-1 marks dysfunctional regulatory T cells in malignant gliomas. JCI Insight. 2016;1(5):e85935.

48. Spitzer MH, et al. Systemic immunity is required for effective cancer immunotherapy. Cell. 2017;168(3):487-502.e15.

49. Kaplan DH, et al. Demonstration of an interferon gamma-dependent tumor surveillance system in immunocompetent mice. Proc Natl Acad Sci USA. 1998;95(13):7556-7561.

50. Calzascia T, et al. TNF-alpha is critical for antitumor but not antiviral T cell immunity in mice. J Clin Invest. 2007;117(12):3833-3845.

51. Kumar V, Patel S, Tcyganov E, Gabrilovich DI. The nature of myeloid-derived suppressor cells in the tumor microenvironment. Trends Immunol. 2016;37(3):208-220.

52. Gabrilovich DI, Ostrand-Rosenberg S, Bronte V. Coordinated regulation of myeloid cells by tumours. Nat Rev Immunol. 2012;12(4):253-268.

53. Zou W. Immunosuppressive networks in the tumour environment and their therapeutic relevance. Nat Rev Cancer. 2005;5(4):263-274.

54. Zaretsky JM, et al. Mutations associated with acquired resistance to PD-1 blockade in melanoma. N Engl J Med. 2016;375(9):819-829.

55. Shin DS, et al. Primary resistance to PD-1 blockade mediated by JAK1/2 mutations. Cancer Discov. 2017;7(2):188-201.

56. Restifo NP, Smyth MJ, Snyder A. Acquired resistance to immunotherapy and future challenges. Nat Rev Cancer. 2016;16(2):121-126. 